\title{
Distributed Local MRF Models for Tissue and Structure Brain Segmentation
}

\author{
Benoit Scherrer, Florence Forbes, Catherine Garbay, Michel Dojat, Senior Member, IEEE
}

\begin{abstract}
Accurate tissue and structure segmentation of Magnetic Resonance (MR) brain scans is critical in several applications. In most approaches this task is handled through two sequential steps. We propose to carry out cooperatively both tissue and subcortical structure segmentation by distributing a set of local and cooperative Markov Random Field (MRF) models. Tissue segmentation is performed by partitioning the volume into subvolumes where local MRFs are estimated in cooperation with their neighbors to ensure consistency. Local estimation fits precisely to the local intensity distribution and thus handles nonuniformity of intensity without any bias field modelization. Similarly, subcortical structure segmentation is performed via local MRF models that integrate localization constraints provided by a priori fuzzy description of brain anatomy. Subcortical structure segmentation is not reduced to a subsequent processing step but joined with tissue segmentation: the two procedures cooperate to gradually and conjointly improve model accuracy. We propose a framework to implement this distributed modeling integrating cooperation, coordination, and local model checking in an efficient way. Its evaluation was performed using both phantoms and real 3T brain scans, showing good results and in particular robustness to nonuniformity and noise with a low computational cost. This original combination of local MRF models, including anatomical knowledge, appears as a powerful and promising approach for MR brain scan segmentation.
\end{abstract}

Index Terms-Markov Random Field, EM estimation, MRI, Human brain

\section{INTRODUCTION}

A UTOMATIC MR brain scan segmentation is a challenging task and has been widely addressed in the last 15 years. Difficulties arise from various sources including the size of the data, the low contrast between tissues, the limitation of available a priori knowledge, local perturbations such as noise or global perturbations such as intensity nonuniformity. Robust-to-noise segmentation is generally addressed via Markov Random Field (MRF) modelization [1]-[8]. This statistical framework introduces local spatial dependencies between voxels, providing a labeling regularization. The intensity

Copyright (c) 2009 IEEE. Personal use of this material is permitted. However, permission to use this material for any other purposes must be obtained from the IEEE by sending a request to pubs-permissions @ ieee.org.

Benoit Scherrer is with the INSERM, U836, Grenoble, F-38043, France, with the CNRS, Laboratoire LIG-Institut IMAG, MAGMA, France and with the Université Joseph Fourier, France (e-mail: benoit.scherrer@imag.fr).

Florence Forbes is with the INRIA, Laboratoire Jean Kuntzmann, MISTIS, France and with the Université Joseph Fourier, France (e-mail: florence.forbes@inrialpes.fr).

Catherine Garbay is with the CNRS, Laboratoire LIG-Institut IMAG, MAGMA, France and with the Université Joseph Fourier, France (e-mail: catherine.garbay@imag.fr).

Michel Dojat is with the INSERM, U836, Grenoble, F-38043, France and with the Université Joseph Fourier, France (e-mail: michel.dojat@ujfgrenoble.fr).

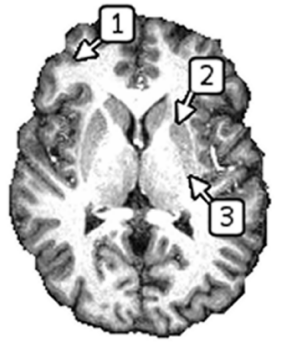

Fig. 1. Illustration of the varying tissue radiometry due to biological tissue properties on a T1-weighted MR scan. The cortex Grey Matter (mark 1) appears darker than the putamen one (marks 2 and mark 3). In addition we observe a tissue nonuniformity inside some structures (see the greylevel difference between mark 2 and mark 3 for instance).

nonuniformity results in spatial intensity variations within each tissue. Even if it does not really affect visual perception, the nonuniformity of image intensity is a major obstacle to an accurate automatic segmentation. It arises on the one hand from MR hardware imperfections, such as magnetic field non uniformity or receiver coil imperfections, and on the other hand from biological tissue properties. In White and Grey matter relaxation time $T_{1}$ and $T_{2}$ vary as a function of magnetic field [9], anatomical regions [10] and age [11]. For instance, cortex ribbon and subcortical structures, such as caudate nucleus, putamen and thalamus, are all composed of Grey Matter (GM) but show a slightly different radiometry in a T1-weighted MR scan due to histology and cellular density variations (see Figure 1).

Most of the proposed approaches share two main characteristics. First, tissue models are estimated globally through the entire volume and then suffer from imperfections at a local level. Second, tissue and structure segmentations are considered as two successive tasks and treated relatively independently. Global tissue segmentation approaches usually introduce explicit so called "bias field" models, which have to be estimated, to account for the intensity nonuniformity due to hardware imperfections [2], [5], [12]-[15]. Bias field models are based on underlying assumptions that are not always valid and generates additional computational burden for their estimation. Intensity nonuniformity due to tissue biological properties is seldom addressed [2], [12]. Local segmentation approaches are attractive alternatives. The principle is to locally compute the tissue intensity models in various subvolumes of the volume. These models better reflect local intensity distributions and are likely to handle different sources of intensity nonuniformity. Existing local tissue segmentation 
approaches either use local estimation as a preprocessing step to estimate a nonuniformity model and then restore the image [16], or use redundant information to ensure consistency and smoothness between local estimated models [17], [18], greedily increasing the computational cost. They however reveal that local estimation is a potentially promising approach to handle intensity nonuniformity for tissue segmentation.

The automatic segmentation of subcortical structures is a challenging task as well. It cannot be performed based only on intensity distributions and requires the introduction of a priori knowledge. This a priori knowledge is classically provided via a global non-rigid atlas registration [19], [20]. Other approaches [21] use statistical local shape modeling and require a training set which may not always be available. A recent alternative approach is to describe brain anatomy with a set of generic fuzzy spatial relations between structures. These relations, such as distance, orientation or symmetry relations, are ubiquitous in natural language descriptions found in neuroanatomy textbooks [22]. They come from anatomical studies about relative spatial position of structures in the brain. Furthermore, relations between brain structures are more stable among individual subjects, and less dependent on the acquisition parameters than the characteristics of the structures themselves. They were introduced for MR brain segmentation in a deformable model [23] or used as a simple mask in a region based approach [24]. For all these segmentation techniques tissue and structure segmentations are considered as two successive tasks although they are clearly linked. First, a structure is composed of a specific tissue. Second, knowledge about structure locations provides valuable information about local intensity distribution for a given tissue. Consequently, tissue and structure segmentations should be rather unified and considered as cooperative procedures which mutually improve.

In this paper, we present an original LOcal Cooperative Unified Segmentation (LOCUS) approach which 1) performs tissue and subcortical structure segmentation by distributing a set of local MRF models through the volume, 2) segments subcortical structures by introducing prior localization constraints into local MRF models and 3) introduces specific cooperation and coordination mechanisms to ensure local model consistency and to link tissue and structure segmentation. More specifically, tissue segmentation is performed by a set of local MRF models distributed in the volume. MRF's parameters are estimated locally in interaction with neighboring MRF models thanks to a new DIstributed Local extension of the EM algorithm (referred to as DILEM). Subcortical structure segmentation is performed by local MRF models that integrate a priori localization constraints based on fuzzy spatial relations. Contrary to other approaches, the fuzzy spatial relations knowledge is not static, but updated and improved during iterations. Subcortical structure segmentation is not reduced to a second subsequent step: tissue segmentation dynamically takes into account the structure knowledge as an a priori in its MRF models, while structure segmentation relies in turn on updated tissue intensity model estimations whose accuracy gradually improves. A priori knowledge is easily integrated using the so called external field rarely considered in MRF modeling. The apparent complexity of LOCUS is well reduced by considering its implementation in a multiagent framework. Each agent computes a local MRF model and cooperates with others for model refinement. Compared to the most used segmentation tools SPM5 and FAST, such an approach shows similar results with shorter computational time. In addition, it appears to be more robust to very high intensity nonuniformity.

This paper is organized as follows. In Section 2, we review existing work and point out the limitations of current global and local tissue segmentation methods and the limitations of structure segmentation approaches. Section 3 presents our LOcal and Cooperative Unified Segmentation approach based on distributed MRF models. Section 4 reports our evaluation performed with the following strategy: first, only tissue segmentation was evaluated, showing good results and in particular robustness to intensity nonuniformity and noise with a low computational cost. Second, we evaluated the cooperative tissue and subcortical structure segmentations. At last, we performed image simulation with a gradually more complex model to exhibit the quality of our modelization. In Section 5, we discuss this original combination of local Markov models which appears to be a powerful and very promising approach for MR brain scan segmentation.

\section{Statistical MR Brain Scan Segmentation}

\section{A. Tissue Segmentation}

We generally consider the segmentation of the brain in three tissues : Cephalo-Spinal Fluid (CSF), Grey Matter (GM) and White Matter (WM). Statistical region-based approaches of tissue segmentation aim at modeling voxel intensities as probability distributions. For T1-weighted MR brain scan, the distribution of each tissue class is commonly modeled as a Gaussian probability density function whose parameters are estimated via an EM [25] or EM-like algorithms. The correction of nonuniformity is a key point to an accurate segmentation. Most approaches then divide in two classes. Global segmentation approaches (Section II-A1) require an explicit nonuniformity model to be estimated. Local segmentation approaches (Section II-A2) rather consider the segmentation problem in a different way that intrinsically handles intensity nonuniformity.

1) Global MR Image Segmentation Approaches with Nonuniformity Correction: Nonuniformity due to tissue biological properties has been seldom addressed in MR image segmentation. In [12] and [2] the use of Parzen-window distributions is proposed to model the conditional probability of intensity for tissue classes. These distributions are trained from a set of representative voxels for each tissue class. These training set must be provided via user interaction, making the segmentation not fully objective and reproducible. On the contrary, correction of nonuniformity due to hardware imperfections has been widely addressed. Some correction methods are applied before or during the image acquisition, and are referred as prospective methods. They aim at calibrating and improving the image acquisition process. Some of them are based on 
the acquisition of a uniform phantom with known physical properties [26], or the use of special MR sequences. For instance, Noterdaeme and Brady [27] have recently proposed to determine inhomogeneities from a series of calibration scans, which are fast to acquire before the image acquisition. We focus in this paper on retrospective methods, which aims at correcting inhomogeneity after the image acquisition. Inhomogeneity is generally modeled as a single slowly varying multiplicative bias field to be estimated and resulting in a so called Bias Field Correction. Two kind of approaches are classically distinguished for retrospective bias field correction: sequential or combined methods.

In sequential methods, bias field correction is performed as a preprocessing step prior to the segmentation step. Low-pass filters or homomorphic filters were typically used to restore the image by removing low-frequency components from the log-transformation of the image. These approaches have the advantage of their simplicity and efficiency in implementation, but are likely to corrupt edges and other high-frequency details of the image. More sophisticated preprocessing approaches have therefore been proposed. Sled et al. [28] propose a nonparametric nonuniform intensity normalization (N3) method which searches for the nonuniformity field to maximize the frequency content of the image intensity distribution. Mangin [29] proposes a method based on entropy minimization, with the aim to unflat the image histogram before the segmentation step. In the PArametric Bias field Correction (PABIC) method, Styner et al. [30] propose an intensity-based approach driven by the assumption that the image is composed of pixels assigned to a small number of classes with a priori known statistics. They correct for the inhomogeneity by "pushing" each pixel intensity to a value which is near to one of the predefined class means. They model the bias field by Legendre polynomials and formulate the estimation as a nonlinear energy minimization problem.

For their part, combined methods estimate tissue classification and bias field correction iteratively, making both estimates gradually more accurate. Wells et al. [12] use a zero mean Gaussian distribution to model a log-transformed bias field. Tissue segmentation and bias field estimation are then performed iteratively in an EM framework [25]. Held et al. [2] introduce additional spatial dependencies between voxels in this model by using MRF models for both label and nonuniformity fields. However, bias field estimation can be significantly affected with such a parametric approach when classes do not follow a Gaussian distribution in the image, like non brain tissues, CSF or pathological regions [14], [15]. Guillemaud and Brady [14] introduce an additional outlier class with a uniform distribution to model these tissues, giving significantly better results. Van Leemput et al. [15] prefer to model bias field as a linear combination of smooth fourthorder polynomial basis functions in a MRF framework. Most of these approaches introduce the log-transformation of the intensity to model the multiplicative bias field as an additive artifact in log-space and make the estimation tractable. This leads to several drawbacks. Numerical problems result from transforming values near zero to the log domain. The nonlinear log-transformation also makes tissue separation more

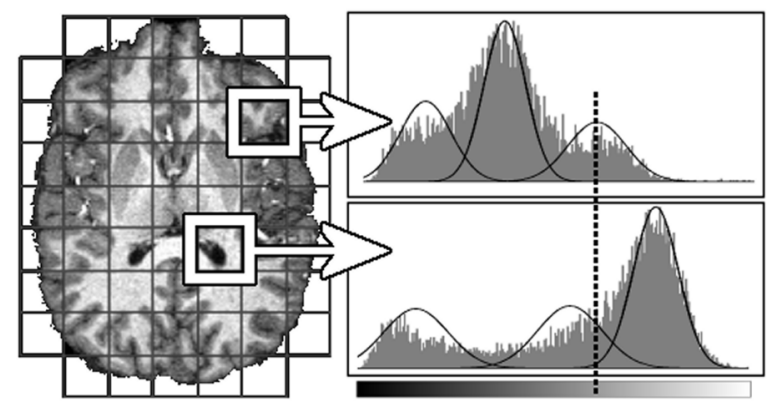

Fig. 2. Variations in local distribution of intensity. The two histograms corresponding to two different subvolumes illustrate the intensity variation for each tissue class. The vertical bar shows a greylevel intensity labeled either as WM or GM depending on the subvolume.

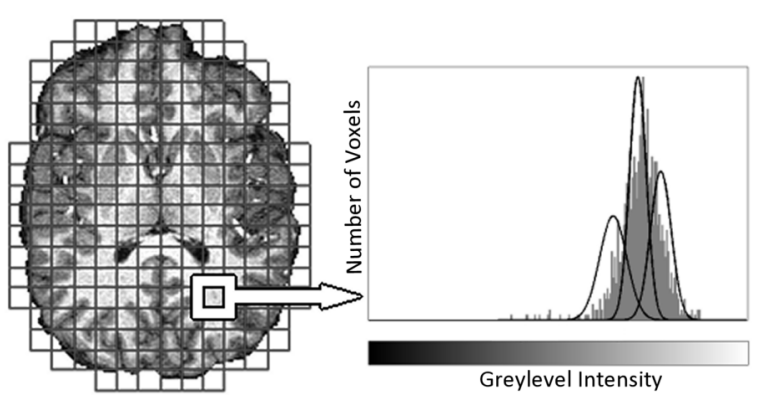

Fig. 3. Importance of subvolume size: in small subvolumes the three tissue classes may not always be all well represented and therefore lead to inaccurate estimation.

difficult and the additive Gaussian noise assumption invalid for the transformed data. Ashburner et al. [13] propose a different model. They keep clear of the log-transformation and include extra parameters that account for the intensity nonuniformity within the mixture of Gaussians. This approach is attractive but relies, as the other approaches, on the unrealistic assumption of a single bias field affecting all tissue classes equally. Marroquin et al. [5] propose to estimate separate smooth parametric models for each class without any log-transformation. This model shows good results but requires the introduction of prior knowledge, provided by a nonrigid atlas registration. Such a step is highly time consuming and could fail in some conditions. A major handicap is that all these approaches estimate global parameters through the whole volume that do not reflect local distribution of intensities.

\section{2) Implicit Nonuniformity Correction via Local approaches:} Local estimation in MR brain scan segmentation has been seldom addressed in the literature, although it seems a good way to handle the main sources of nonuniformity without an explicit bias field estimation. As shown in Figure 2, estimated local models fit more accurately to local image properties. A given greylevel intensity can be labeled either in one class or an other depending on the local subvolume. The size of the subvolumes used to estimate local models is however critical. The larger the size, the more sensitive to nonuniformity is the approach because the estimation is more likely to be perturbed by intensity nonuniformity. Yet, too small partitions may result 
in unreliable model estimations because some tissues are likely to be under-represented in small regions. For example, depending on the location, some subvolumes may contain only white matter as shown in Figure 3. The 3-class segmentation then leads to inaccurate estimation of CSF, GM and WM intensity models. Thus, the two main challenges of local approaches are (1) to allocate a suitable size and shape to local subvolumes and (2) to correct poorly estimated local models and ensure consistency between neighboring models. These difficulties probably explain why only a few local approaches are proposed in the literature.

To handle the first difficulty, regular cubic subvolumes are typically used. For the second, no efficient solution is proposed to date. Grabowski et al. [31] fit Gaussian distributions to local histograms with constraints based on a global distribution fitting to avoid poor model estimations. The approach is then not fully local and the proposed global constraints are not suitable when a high level of intensity nonuniformity is considered. Richard et al. [32] implement a multi-agent approach where local cooperative agents segment their subvolume combining Gaussian mixture modelization and region growing algorithms. The approach essentially lacks a formal representation of agent relations and robust-to-noise efficiency. The AdaptiveMAP (AMAP) approach of [17] estimates distributed MRF models on a 3-D grid of points using partially overlapping subvolumes and uses bilinear interpolation to obtain the intermediate values. Since no consistency check is performed on locally estimated models, subvolumes need to share sufficiently large overlap to ensure reliable estimation. This results in an extremely high computational cost that prevents AMAP from a practicable use. Shattuck et al. [16] estimate local intensity models on a 3-D grid points as well, but with slightly overlapping subvolumes and a 4-step outlier detection. These local estimations are only used as a preprocessing step to estimate a bias field and to restore the image before a global segmentation approach. Zhu et al. [18] propose a MultiContext FCM approach (MCFCM) where FCM algorithm is performed independently in overlapping subvolumes and use information fusion theory to get final membership degrees from all overlapping subvolumes for each voxel. This approach is attractive but may lead to expensive computational cost when introducing spatial voxel dependencies for a robust-tonoise segmentation.

To summarize, when they are not used as a preprocessing step, local approaches use redundant information to ensure consistency and smoothness between contexts, greedily increasing the segmentation computational time. We rather consider that consistency should be ensured via cooperation between local models.

\section{B. Subcortical Structure Segmentation as a Separate Task}

Segmentation of subcortical structures is a fundamental task for various neuroanatomical researches such as brain development or disease progression studies. Yushkevich et al. [33] have recently proposed an efficient user guided method based on deformable models. They provide manual and semiautomatic tools easily accessible to a wide range of users. User

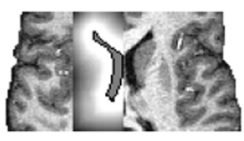

(a)

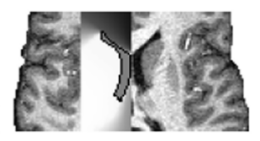

(b)

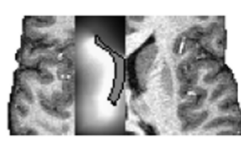

(c)
Fig. 4. Example of fuzzy Maps of Spatial Relations for the left caudate nucleus: (a) expresses "approximately at $5 \mathrm{~mm}$ from the left frontal horn", (b) expresses "in a direction $(-3 \pi / 4, \pi / 2)$ in the Talairach system with respect to the left frontal horn", and (c) is the fusion of the two maps.

guided methods provide good results but are generally timeconsuming and not fully reproducible, introducing inter- and intra-user variability.

Fully automatic segmentation of subcortical structures is a challenging task. The automatic grey nucleus segmentation can not rely only on radiometry information because intensity distributions are largely overlapped. A priori knowledge have to be introduced. Structure segmentation approaches usually rely on a priori atlas describing anatomical structures. The atlas is first warped to the image and then used to label the structures. Two kind of atlases are mainly used: either atlases of labels that directly provide segmentation map after warping [34], or probabilistic atlases that provide a priori knowledge to the segmentation models [20], [35]. In a recent approach, Pohl et al. [19] propose to interleave atlas registration and structure segmentation, gradually improving both procedures. Atlas warping methods are however time consuming and more or less limited due to inter-subject variability. In addition, accurate non-rigid alignment is extremely difficult in low contrast regions, for example near the putamen or near the thalamus.

A recent alternative way to introduce a priori anatomical knowledge is to describe brain anatomy in terms of fuzzy spatial relations between structures. Three kinds of spatial relations are generally considered, namely distance relations, symmetry relations and orientation relations. They are expressed as 3D fuzzy maps [36] to take into account the imprecise nature of the provided knowledge (see Figure 4). Each subcortical structure is described by a set of fuzzy spatial relations with respect to other structures. These relations are provided by a brain anatomist and considered as invariant in human brain anatomy. Fusion operators between fuzzy sets [36] then permit to combine the knowledge provided by each spatial relation and provide a Fuzzy Localization Map (FLM) of the structure in the volume.

Using this approach, Barra et al. [24] first segment tissues as a preprocessing step and then segment structures by considering the FLM as a simple fuzzy mask. They extract voxels from the tissue segmentation map having a membership superior to 0.8 in the FLM. Colliot et al. [23] propose to introduce the knowledge of spatial relations in a deformable model via an external force in its evolution scheme. They propose three different external forces computed from the FLM. Note that to compute the spatial relation of a structure $s_{1}$ with respect to some other structure $s_{2}$, the segmentation of $s_{2}$ must be available. It raises the problem of the root structure required to start the anatomy knowledge construction. One solution is 
to consider the ventricular system that shows a well-defined contrast and is known to be the second largest component of CSF in the brain. This structure is then labeled at first in [23] and [24] using CSF tissue segmentation and some mathematical morphology operators. Then, other structures are hierarchically segmented, first from this reference and then by taking progressively into account previous segmented structures.

All approaches in the literature consider structure segmentation as a task separated from tissue segmentation although it suffers from nonuniformity of tissue intensity as well and from low contrast between regions. In the following section we will show how these two tasks can rather be handled conjointly to mutually improve.

\section{LOCAl COOPERATIVE UNified SEgMENTATION (LOCUS) APPROACH}

We first present the Markovian segmentation framework and emphasize the external field term that allows the introduction of an a priori knowledge in the segmentation. We then describe in Subsection III-B our LOcal Cooperative Unified Segmentation approach for tissue segmentation. In Subsection III-C we show how to integrate the prior localization constraints provided by fuzzy spatial relations in the MRF framework and extend our LOCUS approach to a cooperative tissue and structure segmentation.

\section{A. Markovian Segmentation Framework}

Hidden Markov Random Field Model for Segmentation. Our approach is based on Bayesian analysis which includes the task of segmenting images using probability models. We consider a finite set of $\mathrm{N}$ voxels $V=\{1, \ldots, N\}$ on a regular three-dimensional (3-D) grid. Our aim is to assign each voxel $i$ to one of $K$ classes considering the observed features data $\mathbf{y}=\left(y_{1}, \ldots, y_{N}\right)$. In the case of single MR scan segmentation $y_{i} \in \mathbb{R}$ for all $i \in V$ is the observed grey level intensity at voxel $i$. Both observed intensities and hidden labels are considered to be random fields (collections of random variables) denoted respectively by $\mathbf{Y}=\left\{Y_{1}, \ldots, Y_{N}\right\}$ and $\mathbf{Z}=\left\{Z_{1}, \ldots, Z_{N}\right\}$. Each random variable $Z_{i}$ takes its value in $\left\{e_{1}, \ldots, e_{K}\right\}$ where $e_{k}$ is the $K$-dimensional unit binary vector corresponding to class $k$. Only the $k^{\text {th }}$ component of this vector is non zero and is set to 1 . The small capital notation $\mathbf{z}=\left\{z_{1}, \ldots, z_{N}\right\}$ is used to denote a configuration of $\mathbf{Z}$ corresponding to a realization of the random field. The observed image $\mathbf{Y}$ can be seen as a degraded version of $\mathbf{Z}$. The image $\mathbf{Y}$ depends on $\mathbf{Z}$ through a known conditional probability density function $p\left(\mathbf{y} \mid \mathbf{z}, \Phi_{y}\right)$ which incorporates the image formation model and the noise model and depends on some parameters $\Phi_{y}$. To segment the observed image $\mathbf{Y}$ is to propose an estimate $\hat{\mathbf{Z}}$ of $\mathbf{Z}$ on the basis of $\mathbf{Y}$. Bayesian image segmentation methods are based on the following principles. The unknown segmentation $\mathbf{z}$ is supposed to be a realization of a random field with distribution $p\left(\mathbf{z} \mid \Phi_{z}\right)$ depending on some parameters $\Phi_{z}$. Then the segmentation $\hat{\mathbf{z}}$ is based on the posterior density of $\mathbf{z}$ which can be derived using the Bayes rule as

$$
p(\mathbf{z} \mid \mathbf{y}, \Phi) \propto p\left(\mathbf{y} \mid \mathbf{z}, \Phi_{y}\right) p\left(\mathbf{z} \mid \Phi_{z}\right)
$$

where $\Phi=\left(\Phi_{y}, \Phi_{z}\right)$. A standard segmentation criterion consists of maximizing this density, leading to the Maximum A Posteriori (MAP) estimate of $\mathbf{z}$.

One of the most popular modeling assumptions is to consider the image $\mathbf{z}$ as being a realization of a discrete Markov Random Field. The Markovian segmentation framework [1] is commonly used for MR brain scan segmentation [2]-[8]. It introduces spatial dependencies between voxels, providing a robust-to-noise segmentation. More specifically, $V$ is associated to a neighborhood system where $\mathcal{N}(i)$ denotes the set of voxels neighboring voxel $i$ and the hidden data $\mathbf{Z}$ are described by a Random Field whose probability distribution satisfies the following properties:

$$
\begin{array}{cc}
\forall \mathbf{z}, & p\left(z_{i} \mid \mathbf{z}_{V \backslash\{i\}},\right)=p\left(z_{i} \mid z_{j}, j \in \mathcal{N}(i)\right) \\
\forall \mathbf{z}, & p(\mathbf{z})>0,
\end{array}
$$

where $\mathbf{z}_{V \backslash\{i\}}$ denotes a realization of the field restricted to $V \backslash\{i\}=\{j \in V, j \neq i\}$. Property (2) means that the interactions between site $i$ and the other sites are reduced to interactions with its neighbors, introducing spatial dependencies between voxels. This property plus the positivity property (3) are sufficient for the Hammersley-Clifford theorem to hold. This theorem states that the probability distribution of a Markov field which depends on some parameters $\Phi_{z}$ is a Gibbs distribution given by:

$$
p\left(\mathbf{z} \mid \Phi_{z}\right)=W_{\Phi_{z}}^{-1} \exp \left(-H\left(\mathbf{z} \mid \Phi_{z}\right)\right),
$$

where $H\left(\mathbf{z} \mid \Phi_{z}\right)$ is called the energy function and $W_{\Phi_{z}}=$ $\sum_{\mathbf{z}^{\prime}} \exp \left(-H\left(\mathbf{z}^{\prime} \mid \Phi_{z}\right)\right)$ is a normalization constant. When assuming in addition that the observations $\mathbf{Y}$ are conditionally independent given $\mathbf{Z}$, then $p\left(\mathbf{y} \mid \mathbf{z}, \Phi_{y}\right)$ can be written as a product $p\left(\mathbf{y} \mid \mathbf{z}, \Phi_{y}\right)=\prod_{i \in V} p\left(y_{i} \mid z_{i}, \Phi_{y}\right)$. Using (1), it follows that the conditional probability $p(\mathbf{z} \mid \mathbf{y}, \Phi)$ also corresponds to a Markov field with energy function given by:

$$
H(\mathbf{z} \mid \mathbf{y}, \Phi)=H\left(\mathbf{z} \mid \Phi_{z}\right)-\sum_{i \in V} \log p\left(y_{i} \mid z_{i}, \Phi_{y}\right) .
$$

This energy is composed of two terms:

- The first term of (4) is a regularization term that accounts for spatial dependencies between voxels. In this work we will consider a Potts model with external field. In the following, the ${ }^{t}$ exponent denotes the transpose operator and for two vectors $z_{i}$ and $v_{i},{ }^{t} z_{i} v_{i}$ denotes the scalar product:

$$
H\left(\mathbf{z} \mid \Phi_{z}\right)=-\sum_{i \in V}\left[{ }^{t} z_{i} v_{i}+\frac{\eta}{2} \sum_{j \in \mathcal{N}(i)} V_{i j}\left(z_{i}, z_{j}\right)\right]
$$

where $V_{i j}\left(z_{i}, z_{j}\right)$ introduces spatial interactions between voxels $i$ and $j$. It is classically defined by $V_{i j}\left(z_{i}, z_{j}\right)=$ ${ }^{t} z_{i} z_{j}$ so that $V_{i j}\left(z_{i}, z_{j}\right)=1$ when $z_{i}$ and $z_{j}$ are in the same class and 0 otherwise. Other approaches introduce voxel interactions weighted by their distance. $\eta$ is a parameter that accounts for the strength of spatial interaction. When $\eta$ is positive this model gives higher probability to neighbors that are in the same class. The $v_{i}$ 's are additional parameters. They are K-dimensional 
vectors defining the so-called external field. Each $v_{i}$ can be related to weights accounting for the relative importance of the $K$ classes at site $i$. They can then be used to favor a class at each voxel location. For example $v_{i}={ }^{t}[1,0, \ldots, 0]$ will favor class 1 for voxel $i$. Note that the negation in (5) is due to the negative notation in the Gibbs distribution. The introduction of these extra parameters in the standard Potts model is seldom considered in MRF approaches although they can be estimated as $\eta$. In the following developments, we will not estimate them but show how they can be used to integrate additional a priori knowledge for each voxel in the segmentation procedure. In this case the unknown parameters are reduced to $\Phi_{z}=\{\eta\}$.

- The second term of (4) is the data-driven term based on intensities. For MRI we generally consider a Gaussian probability density function for each tissue class. It follows that for each class $k, p\left(y_{i} \mid z_{i}=e_{k}, \Phi_{y}\right)=$ $g_{\mu_{k}, \sigma_{k}}\left(y_{i}\right)$ where $g_{\mu_{k}, \sigma_{k}}$ denotes the Gaussian distribution with mean $\mu_{k}$ and variance $\sigma_{k}^{2}$. It follows that $\Phi_{y}=\left\{\mu_{k}, \sigma_{k}, k=1 \ldots K\right\}$.

Estimation of MRF.

Segmentation is then performed according to the Maximum A Posteriori principle (MAP) by maximizing over $\mathbf{z}$ the probability:

$$
p(\mathbf{z} \mid \mathbf{y}, \Phi)=W_{\mathbf{y}, \Phi}^{-1} \exp (-H(\mathbf{z} \mid \mathbf{y}, \Phi)),
$$

where $W_{\mathbf{y}, \Phi}^{-1}$ is the corresponding normalization constant. This requires the estimation of the unknown parameters $\Phi$. A standard approach is to use the ICM algorithm [37] that alternates between parameter estimation and segmentation. A valid segmentation is computed at each iteration and then prevents from propagating segmentation uncertainties. The resulting parameters estimates are then likely to be biased. As an alternative, we rather consider EM-based algorithms and use variants proposed by Celeux et al. [38]. They are based on Mean-field like approximations to make the MRF model case tractable. In a segmentation context, they provide parameters estimation $\hat{\Phi}$ but also values for missing data by providing membership probabilities to each class. The rule is that observation $y_{i}$ is assigned to class $\hat{k}$ if $\hat{k}=\arg \max _{k} p\left(Z_{i}=e_{k} \mid y, \hat{\Phi}\right)$. As specified below, in a MRF setting such posterior probabilities $p\left(Z_{i}=e_{k} \mid y, \hat{\Phi}\right)$ are not tractable so that the membership probabilities computed in the Mean Field like algorithms are approximations of these posterior probabilities. The details are given in [38].

Assuming $\Phi$ unknown, the aim is to get the maximum likelihood estimate of this parameter knowing the observations $\mathbf{y}$. The log-likelihood of the model is

$$
L(\Phi)=\log p(\mathbf{y} \mid \Phi)=\log \sum_{\mathbf{Z}} p(\mathbf{y}, \mathbf{z} \mid \Phi) .
$$

The EM algorithm [25] is an iterative algorithm aimed at maximizing over $\Phi$ this log-likelihood by maximizing at iteration $q$ the expectation of the complete log-likelihood knowing the observation $\mathbf{y}$ and a current estimate $\Phi^{(q)}$,

$$
Q\left(\Phi \mid \Phi^{(q)}\right)=\mathbb{E}_{\Phi^{(q)}}[\log p(\mathbf{y}, \mathbf{Z} \mid \Phi) \mid \mathbf{Y}=\mathbf{y}] .
$$

The EM algorithm can therefore be described as follows,

(1) start from an initial guess $\Phi^{(0)}$ for $\Phi$,

(2) update the current estimate $\Phi^{(q)}$ to

$$
\Phi^{(q+1)}=\arg \max _{\Phi} Q\left(\Phi \mid \Phi^{(q)}\right) .
$$

The updating part (2) can be divided into two steps. The computation of $Q\left(\Phi \mid \Phi^{(q)}\right)$ corresponds to the $\mathrm{E}$ (expectation) step and the maximization with respect to $\Phi$ to the $\mathrm{M}$ (maximization) step. There are two difficulties in evaluating $Q$ in this case. Both the partition function $W_{\Phi_{z}}$ and the conditional probabilities, $p\left(z_{i} \mid \mathbf{y}, \Phi^{(q)}\right)$ and $p\left(z_{i}, z_{j} \mid \mathbf{y}, \Phi^{(q)}\right)$ for $j$ in the neighborhood $\mathcal{N}(i)$ of $i$, cannot be computed exactly. For MRF models, due to the dependence structure, the exact EM is not tractable and approximations are required. In this paper we use some of the approximations presented in [38]. These approximations are based on the mean field principle which consists in replacing the intractable Markov distributions by factorized ones for which the exact EM can be carried out. This allows to take the Markovian structure into account while preserving the good features of EM. Celeux et al. [38] generalized the mean field principle and introduced different factorized models resulting in different procedures. Briefly, these algorithms can be presented as follows. Informally, the mean field approach consists in approximating the intractable probabilities by neglecting fluctuations from the mean in the neighborhood of each voxel $i$. More generally, we talk about mean field-like approximations when the value for voxel $i$ does not depend on the value for other voxels which are all set to constants (not necessarily the means) independently of the value for voxel $i$. These constant values are not arbitrary but satisfy some appropriate consistency conditions. In particular the mean-field, mode-field and simulated-field algorithms in [38] consist in three different ways of setting the constants.

\section{B. LOCUS-T approach for Tissue Segmentation.}

1) Markov Modeling: We consider a regular cubic partitioning of the volume $V$ in a number of $C$ non-overlapping subvolumes $\left\{V_{c}^{T}, c \in 1 \ldots C\right\}$ :

$$
\left\{\begin{array}{l}
\forall c \neq c^{\prime}, V_{c}^{T} \cap V_{c^{\prime}}^{T}=\emptyset \\
\bigcup_{c=1}^{C} V_{c}^{T}=V
\end{array} .\right.
$$

Each subvolume $V_{c}^{T}$ is segmented with a local MRF model $M_{c}^{T}$ that cooperates with neighboring local MRF as described in the following subsections. We consider $K=3$ tissue classes: $C S F$ (Cephalo-Spinal Fluid), GM (Grey Matter) and $W M$ (White Matter). We denote by $t=\left\{t_{i}, i \in V_{c}^{T}\right\}$ the hidden tissue classes at each voxel. The $t_{i}$ 's take their values in $\left\{e_{1}, e_{2}, e_{3}\right\}$ respectively for classes $\left\{e_{C S F}, e_{G M}, e_{W M}\right\}$. Each local MRF model $M_{c}^{T}$ is defined by the Gibbs distribution of energy (see Equations 4 and 5):

$$
\begin{aligned}
H^{c}\left(\mathbf{t} \mid \mathbf{y}, \Phi^{c}\right)= & -\sum_{i \in V_{c}^{T}}\left[{ }^{t} t_{i} \lambda_{i}^{c}+\frac{\eta^{c}}{2} \sum_{j \in \mathcal{N}(i)} V_{i j}\left(t_{i}, t_{j}\right)\right. \\
& \left.+\log p\left(y_{i} \mid t_{i}, \Phi_{y}^{c}\right)\right],
\end{aligned}
$$


where the unknown parameters are $\Phi^{c}=\left\{\Phi_{t}^{c}, \Phi_{y}^{c}\right\} . \Phi_{t}^{c}=$ $\left\{\eta^{c}\right\}$ accounts for the local strength of spatial interaction, while $\Phi_{y}^{c}$ are the estimated parameters of the local Gaussian tissue intensity models. The external field parameters denoted by $\left\{\lambda_{i}^{c}, i \in V_{c}^{T}\right\}$ are not estimated but used to incorporate information coming from structure segmentation (see Section II-B).

The MRF model $M_{c}^{T}$ introduces spatial dependencies between voxels in its subvolume $V_{c}^{T}$, providing a consistency of neighboring labels. As mentioned in Subsection II-A2, local approaches may lead to poor model estimation if a class is under-represented in a local subvolume (see Figure 3). It is then required to check the reliability of a local model and to correct it if needed. Instead of introducing global constraints as in [16] we propose to introduce local constraints based on the neighborhood of each local MRF, providing a consistency of neighboring local models. It then introduces an additional level of regularization to the standard label regularization provided by MRF.

2) Cooperation between neighboring MRF models: We define a neighborhood system between local MRF models. We denote by $\mathcal{N}\left(M_{c}^{T}\right)$ the set of MRF models neighboring $M_{c}^{T}$ and by $d\left(c, c^{\prime}\right)$ the Euclidian distance between the centers of the subvolumes $V_{c}^{T}$ and $V_{c^{\prime}}^{T}$. The MRF $M_{c}^{T}$ cooperates with its neighbors $\mathcal{N}\left(M_{c}^{T}\right)$ to ensure a global consistency of the local estimated model: it performs model checking, model correction and model interpolation as described below.

\section{Model Checking}

We compute for each $M_{c}^{T}$ a model $\widetilde{M_{c}^{T}}$ averaging the models of $\mathcal{N}\left(M_{c}^{T}\right)$, given by:

$$
\forall k=1 . .3\left\{\begin{array}{r}
\widetilde{\mu_{k}^{c}}=D^{-1} \sum_{c^{\prime} \in \mathcal{N}\left(M_{c}^{T}\right) \frac{\mu_{k}^{c^{\prime}}}{d\left(c, c^{\prime}\right)},} \\
\widetilde{\sigma_{k}^{c}}=D^{-1} \sum_{c^{\prime} \in \mathcal{N}\left(M_{c}^{T}\right) \frac{\sigma_{k}^{\prime}}{d\left(c, c^{\prime}\right)},} \\
\text { with } \quad D=\sum_{c^{\prime} \in \mathcal{N}\left(M_{c}^{T}\right)} d^{-1}\left(c, c^{\prime}\right) .
\end{array}\right.
$$

Then, for each class $k$, we compare intensity models of $M_{c}^{T}$ and $\widetilde{M_{c}^{T}}$ using the Kullback-Leibler distance given by:

$$
K L\left(g_{\mu_{1}, \sigma_{1}}, g_{\mu_{2}, \sigma_{2}}\right)=\frac{\left(\sigma_{1}^{2}-\sigma_{2}^{2}\right)^{2}+\left(\mu_{1}-\mu_{2}\right)^{2}\left(\sigma_{1}^{2}+\sigma_{2}^{2}\right)}{4 \sigma_{1}^{2} \sigma_{2}^{2}}
$$

Thus, $\mathcal{D}_{k}^{c}=K L\left(g_{\mu_{k}^{c}, \sigma_{k}^{c}}, g_{\widetilde{\mu_{k}^{c}}, \sigma_{k}^{c}}\right)$ provides a measure of dissimilarity between the two intensity models of class $k$.

\section{Model Correction}

We assume that for each tissue class $k$ the model $M_{c}^{T}$ is likely to be close to the local mean model $\widetilde{M_{c}^{T}}$. This assumption is consistent with the slowly varying property of nonuniformities. We compute the corrected mean $\widehat{\mu_{k}^{c}}$ and variance $\widehat{\sigma_{k}^{c}}$ of class $k$ from a linear combination of intensity models in $M_{c}^{T}$ and $\widetilde{M_{c}^{T}}$ :

$$
\begin{aligned}
& \widehat{\mu_{k}^{c}}=(1-\kappa) \mu_{k}^{c}+\kappa \widetilde{\mu_{k}^{c}} \\
& \widehat{\sigma_{k}^{c}}=(1-\kappa) \sigma_{k}^{c}+\kappa \widetilde{\sigma_{k}^{c}},
\end{aligned}
$$

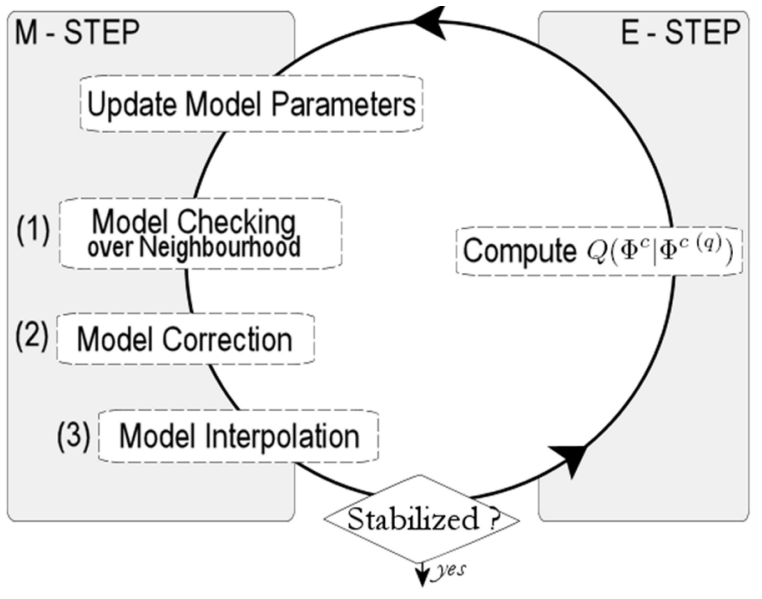

Fig. 5. DIstributed Local EM (DILEM) Algorithm for LOCUS-T. The loop without the steps (1), (2) and (3) corresponds to the classical EM algorithm.

with $\kappa \in[0,1]$. We define two thresholds $\delta_{\text {keep }}$ and $\delta_{\text {replace }}$ with $\delta_{\text {keep }} \leq \delta_{\text {replace }}$ and propose the following strategy of model correction:

- If $\mathcal{D}_{k}^{c} \leq \delta_{k e e p}$, the estimated local model of class $k$ is considered correct and we keep it so that $\kappa=0$

- If $\mathcal{D}_{k}^{c}>\delta_{\text {replace }}$ we replace the estimated model by the local mean model of class $k$ so that $\kappa=1$.

- Else, if $\delta_{\text {keep }}<\mathcal{D}_{k}^{c} \leq \delta_{\text {replace, }}$, we partially correct the estimated model with a linear interpolation: $\kappa=$ $\frac{\mathcal{D}_{k}^{c}-\delta_{\text {keep }}}{\delta_{\text {replace }}-\delta_{\text {keep }}}$

Model Interpolation

Model correction provides one corrected tissue intensity model, namely three Gaussians, for $M_{c}^{T}$. Then we compute one intensity model per voxel by using cubic splines interpolation between corrected models of $M_{c}^{T}$ and of $\mathcal{N}\left(M_{c}^{T}\right)$. We used the kriging method [39] that was firstly introduced for geology and mining applications. Kriging is a general statistical interpolation framework that can be equivalent to cubic splines interpolation in some conditions. The main advantage of this method is its natural extensibility to the 3dimensional interpolation and its computational efficiency. It determines the weights of the contribution of each intensity models via the resolution of a simple linear system.

It follows that $\Phi_{y}^{c}=\left\{\mu_{k, i}, \sigma_{k, i}, k=1 \ldots K, i \in V_{c}^{T}\right\}$. This results in a non-stationary field-like approach. There is not a unique mixture of Gaussians describing the intensity distribution in $V_{c}^{T}$ but one mixture of Gaussians per voxel. It has the advantage 1) to ensure smooth model variation between neighboring subvolumes and 2) to intrinsically handle nonuniformity of intensity inside each subvolume. In the following, since subvolumes are not overlapping, we will denote by $\Phi_{i y}=\left\{\mu_{k, i}, \sigma_{k, i}, k=1 \ldots K\right\}$ the parameters of the mixture of Gaussians for voxel $i \in V$.

Resulting parameters estimation algorithm

We introduce these three steps in the MRF estimation procedure. It can be introduced either in ICM or in any of the different EM-based algorithms proposed by [38]. Figure 5 
shows the introduction of these three steps in an EM-like algorithm by modifying the M-Step. In the following, we refer to this algorithm as the DIstributed Local EM (DILEM) algorithm. Note that the $\eta^{c}$ parameter is neither checked nor corrected since it is not estimated but considered as the inverse of a decreasing temperature (see Section IV-A). At convergence, the value of $\eta^{c}$ is then the same for all subvolumes.

3) Coordination between local EM procedures: Rather than launching all local EM procedures simultaneously, we consider some heuristics to order their execution and ensure a robust segmentation. First each EM is locally initialized. To improve robustness, initial models are computed on the information available in a larger subvolume than the MRF subvolume. We choose to compute this initialization on a territory 1) centered on the MRF subvolume and 2) with a size twice the size of the MRF subvolume. Global intensity models are then computed on the entire volume using a standard non spatial EM algorithm. Only the local EM procedures with initial models closest to the global model are first activated, providing reliable references to their neighbors. When a local EM procedure is activated, it performs model checking and model correction in its DILEM loop, considering all neighboring MRF models regardless of their current state. When a local EM procedure is stabilized, its neighbors are activated to perform estimation in turn. Two cases are possible after the estimation procedure activation of a model $M_{c}^{T}$ :

- If the estimation procedure of $M_{c}^{T}$ has never been launched yet, it is just launched.

- If the estimation procedure of $M_{c}^{T}$ has already reached a convergence state, model checking is performed. When model correction is required, the corresponding EM procedure is restarted to take into account the model modifications. We define a maximum number $M A X_{\text {tissue_estimation_restart }}$ of estimation procedure executions to ensure that the algorithm stops.

LOCUS-T then estimates local intensity models in subvolumes and introduces two levels of regularization in the segmentation procedure: a regularization of labels via MRF models and a regularization of local estimated models. The local estimation procedures are asynchronously scheduled via interactions between models. The more reliable subvolumes, for which the local model is closest to the global model, are considered first and coordination mechanisms then ensure the information diffusion over subvolumes.

\section{LOCUS-TS Approach For Cooperative Tissue and Struc- ture Segmentation.}

We extend our LOCUS approach to tissue and subcortical structure segmentation, introducing anatomical knowledge in our model. We consider $L$ subcortical structures and denote by $\mathbf{s}=\left\{s_{1}, \ldots, s_{N}\right\}$ the structure hidden classes. The $s_{i}$ 's take their value in $L+1$ classes for the $L$ structures and the background. A priori knowledge required for structure segmentation is provided by the fuzzy description of the brain anatomy described in subsection II-B. The resulting Fuzzy
Localization Map (FLM) $f^{l}$ of the structure $l$ is used in two ways:

- it provides a region of interest containing the structure $l$. We compute the subvolume $V_{l}^{S}$ of structure $l$ as the rectangular bounding box containing the voxels whose value in the FLM $f^{l}$ is higher than a given threshold $\delta_{\text {ROI }}$. We denote by $N^{l}$ the number of voxels in $V_{l}^{S}$.

- it is used as an a priori anatomical knowledge to segment the structure $l$.

1) Markov Modeling: A natural approach to label the $L$ subcortical structures is to define a unique global model with $L+1$ classes. The hidden tissue classes $s_{i}$ take their value in $\left\{e_{0}, e_{1}, \ldots, e_{L}\right\}$ for voxels of the background $\left(s_{i}=e_{0}\right)$ or voxels belonging to the structure $l\left(s_{i}=e_{l}\right)$. We then decompose this global model into $L$ local binary Markov models $M_{l}^{S}$. Each $M_{l}^{S}$ labels voxels of its subvolume $V_{l}^{S}$ in $K=2$ classes, referred as structure $\left(e_{S}\right)$ and background $\left(e_{B}\right)$. The hidden class $s_{i}^{l}$ is then defined for $i \in V_{l}^{S}$ by:

$$
\begin{cases}s_{i}^{l}=e_{B}=\left[\begin{array}{l}
1 \\
0
\end{array}\right] & \text { if } s_{i} \neq e_{l}, \\
s_{i}^{l}=e_{S}=\left[\begin{array}{l}
0 \\
1
\end{array}\right] & \text { if } s_{i}=e_{l} .\end{cases}
$$

The energy function of the corresponding MRF model $M_{l}^{S}$ associated to structure $l$ is given by:

$$
\begin{aligned}
H^{l}\left(\mathbf{s}^{l} \mid \mathbf{y}, \Psi^{l}\right)= & -\sum_{i \in V_{l}^{S}}\left[{ }^{t} s_{i}^{l} \alpha_{i}^{l}+\frac{\eta^{l}}{2} \sum_{j \in \mathcal{N}(i)} V_{i j}\left(s_{i}^{l}, s_{j}^{l}\right)\right. \\
& \left.+\log p\left(y_{i} \mid s_{i}^{l}, \Psi_{y}^{l}\right)\right],
\end{aligned}
$$

with $\Psi_{s}^{l}=\left\{\eta^{l}\right\}$ and $\Psi^{l}=\left\{\Psi_{s}^{l}, \Psi_{y}^{l}\right\}$. Each structure being composed of a single tissue $T^{l} \in\left\{e_{C S F}, e_{G M}, e_{W M}\right\}$, we do not estimate intensity model of class structure and class background but rather compute these models from tissue intensity models $\Phi_{y}^{c}$ estimated by tissue segmentation (See Subsection III-C3). The external field denoted by $\left\{\alpha_{1}^{l} \ldots \alpha_{N_{l}}^{l}\right\}$, where $\alpha_{i}^{l}$ is a 2-dimensional vector, allows to incorporate the a priori knowledge of the FLM as described below.

2) Integration of prior localization constraints in the $M R F$ Framework: We denote by $f_{i}^{l}$ the value of the FLM $f^{l}$ at voxel $i$ and propose the following external field:

$$
\alpha_{i}^{l}=\gamma_{i}\left[\begin{array}{c}
-\log \left(1-f_{i}^{l}\right) \\
-\log f_{i}^{l}
\end{array}\right],
$$

where $\gamma_{i}>0$ adjusts the influence of the external field. When $f_{i}^{l} \approx 0$, the voxel $i$ is unlikely to belong to the structure. Denoting by $\alpha_{i}^{l}(1)$ (respectively $\alpha_{i}^{l}(2)$ ) the first (respectively the second) component of vector $\alpha_{i}^{l}$, this implies $\alpha_{i}^{l}(1)<$ $\alpha_{i}^{l}(2)$, which favors in (8) the background class. When $f_{i}^{l} \approx 1$, the voxel $i$ is likely to belong to the structure. In that case $\alpha_{i}^{l}(1)>\alpha_{i}^{l}(2)$ and the structure class is favored. We thus propose to introduce the prior fuzzy knowledge of the FLM as relative potentials for each voxel $i$ in the MRF models, weighting the relative importance of each class. 


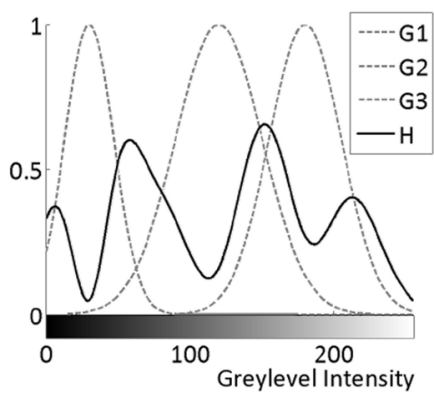

(a)

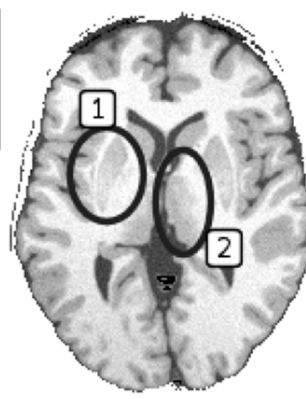

(b)

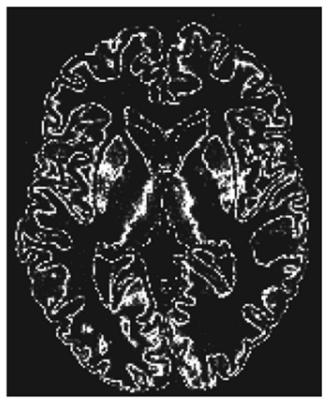

(c)

Fig. 6. Image (a) illustrates the entropy $\mathrm{H}$ for a mixture of three Gaussians G1, G2 and G3. Image (b) is a transversal brain slice and image (c) the corresponding entropy map of the tissue posterior distribution probability (high value are white). In the region of the putamen (mark 1) and the thalamus (mark 2), the entropy is larger because of the low contrast between tissues, leading to an unstable labeling.

The contrast between tissues is low on MR brain scan, specifically inside putamen or thalamus where the intensity of the GM is brighter because of a higher myelin fiber density. In consequence a number of voxels verify $p\left(y_{i} \mid t_{i}=e_{G M}, \Phi_{y}\right) \approx p\left(y_{i} \mid t_{i}=e_{W M}, \Phi_{y}\right)$ which is likely to lead to $p\left(t_{i}=e_{G M} \mid y_{i}, \Phi\right) \approx p\left(t_{i}=e_{W M} \mid y_{i}, \Phi\right)$, making the labeling unstable. This means that in such cases, tissue information based on radiometry is not enough to adequately separate structures. The distribution of the posterior probabilities carries information on the labeling stability. If one distinct class shows a very high probability and all other classes have a low probability, this signifies a stable labeling. When all classes have quasi equal probabilities, the labeling is very unstable. This information is captured in the entropy of the estimated posterior probabilities $H_{i}$ :

$$
H_{i}=-\sum_{k=1 . . K} p\left(t_{i}=e_{k} \mid y_{i}, \Phi\right) \log \left(p\left(t_{i}=e_{k} \mid y_{i}, \Phi\right)\right) \text {. }
$$

It is larger when two tissue probabilities are almost equal, as in some parts of the putamen and the thalamus (see Figure 6). The external field influence $\gamma_{i}$ is then chosen to be stronger depending on this entropy at voxel $i$ via an increasing function $h$ of $H_{i}: \gamma_{i}=h\left(H_{i}\right)$.

3) Cooperation between models: In our approach, tissue and structure segmentations are performed by local models spatially distributed over the volume. Instead of considering these models separately, we propose to link them and to ensure consistency via cooperation mechanisms. We identify cooperation between structure models and cooperation between tissue and structure models. We then define:

- the set $\mathcal{C}_{T \rightarrow S}(l)=\left\{c, V_{c}^{T} \cap V_{l}^{S} \neq \emptyset\right\}$ corresponding to tissue models cooperating with the structure model $M_{l}^{S}$,

- the set $\mathcal{C}_{S \rightarrow T}(c)=\left\{l, V_{l}^{S} \cap V_{c}^{T} \neq \emptyset\right\}$ corresponding to structure models cooperating with the local tissue model $M_{c}^{T}$, and

- the set $\mathcal{C}_{S \rightarrow S}(l)$ corresponding to structure models using $l$ as a reference to construct a spatial relation.

Updating structure segmentation models via tissue segmentation models.

Each structure $l$ being composed of a single tissue $T^{l}$ we naturally base structure intensity models on tissue intensity models estimated in tissue segmentation. More specifically we use tissue intensity models coming from $\mathcal{C}_{T \rightarrow S}(l)$. The structure intensity model for class $e_{S}$ is straightforwardly the tissue intensity model for class $T^{l}$ :

$$
p\left(y_{i} \mid s_{i}^{l}=e_{S}, \Psi_{y}^{l}\right)=p\left(y_{i} \mid t_{i}=T^{l}, \Phi_{y}\right) .
$$

The background class intensity model is then set to a mixture of all tissue intensity models. The rationale is that in model $M_{l}^{S}$, a voxel is in the background class if it is either a voxel belonging to another structure $l^{\prime} \neq l$ or a voxel belonging to none of the $L$ structures. Therefore such a voxel can be in any of the three tissue classes. However, the proportions of the mixture can be determined using the other fuzzy maps and the current tissue segmentation (see details in Appendix).

Feedback of Structure Segmentation on Tissue Segmentation. Conversely, results from structure segmentation are integrated in the tissue segmentation model via the 3-dimensional external field $\lambda_{i}^{c}={ }^{t}\left[\lambda_{i}^{c}\left(e_{C S F}\right), \lambda_{i}^{c}\left(e_{G M}\right), \lambda_{i}^{c}\left(e_{W M}\right)\right]$ of the local tissue MRF $M_{c}^{T}$ (see Equation (7)). More specifically, for voxels belonging to at least one structure, we use for the tissue class $e_{k}$ the weight:

$$
\lambda_{i}^{c}\left(e_{k}\right)=\log \left(\max _{l \in \mathcal{C}_{S \rightarrow T}(c), T^{l}=e_{k}} p\left(s_{i}^{l}=e_{S} \mid \mathbf{y}, \Psi^{l}\right)\right),
$$

so that the tissue class $e_{k}$ will be favored when voxel $i$ belongs to a structure composed of the tissue $e_{k}$. For the other voxels we set $\lambda_{i}^{c}={ }^{t}[0,0,0]$.

4) Coordination between models: As in LOCUS-T, we introduce coordination mechanisms to schedule the estimation of the local models.

- Activation of structure segmentation: as in [23] and [24] we consider the ventricular system to start the construction of the fuzzy spatial relations describing the anatomy knowledge. Denoting by $l=1$ the ventricular system index, the estimation procedure of $M_{l=1}^{S}$ waits for all tissue estimation procedures of $\mathcal{C}_{T \rightarrow S}(1)$ to be stabilized to segment a first version of the ventricular system via mathematical morphology operators and CSF segmentation. 


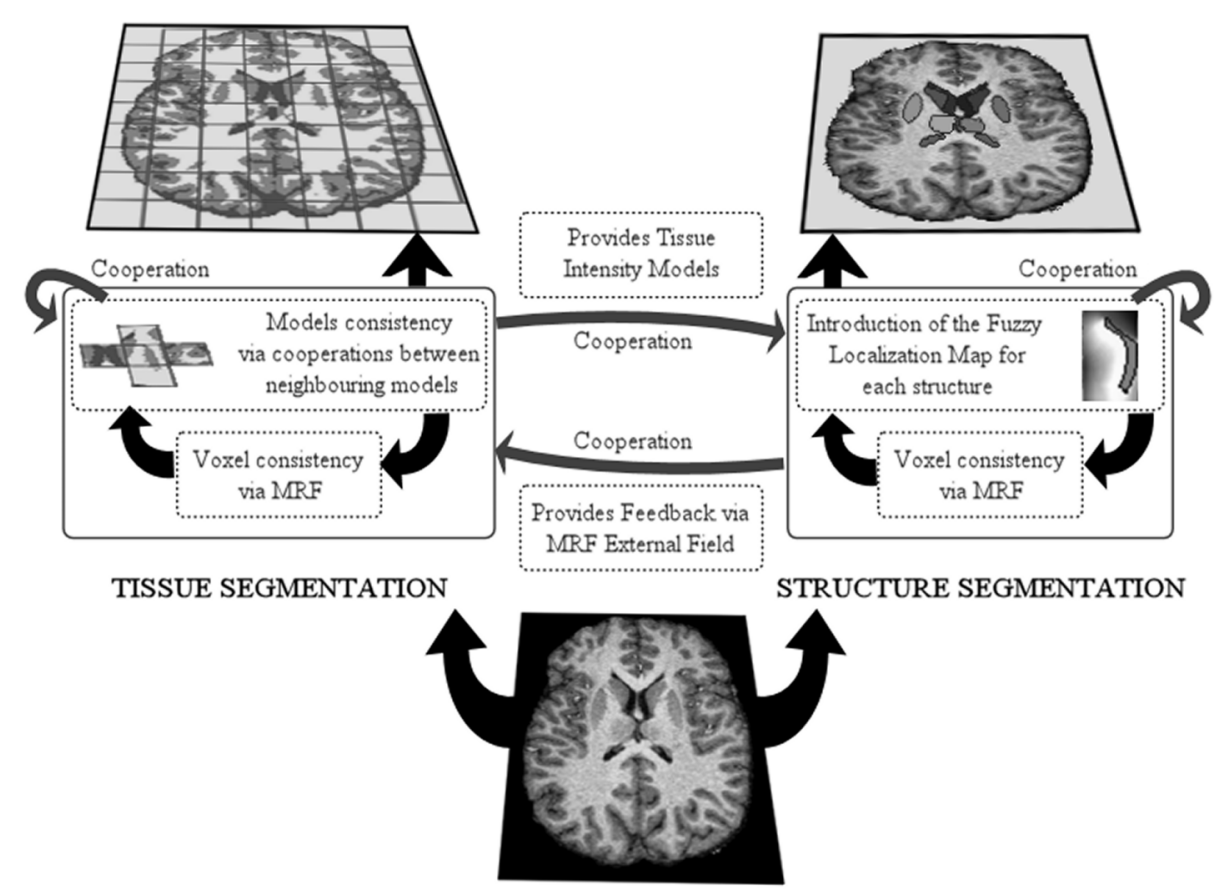

Fig. 7. Synthetic view of our LOCUS approach for combined tissue and structure segmentation.

- Updating Fuzzy Maps: when the segmentation of structure $l$ is updated the structure models of $\mathcal{C}_{S \rightarrow S}(l)$ take it into account by re-computing the maps corresponding to spatial relations with respect to $l$, making the knowledge gradually more accurate. Only fuzzy spatial relation maps describing adjacent structures are updated at each segmentation update. Other relation maps are updated only when the segmentation of the reference structure has sufficiently changed. The threshold is based on the relative number of voxels modified. This parameter is not sensitive due to the general nature of the spatial relation knowledge.

- Updating Tissue Estimation: each time the convergence of a structure segmentation algorithm is reached, it activates the corresponding cooperative tissue estimation procedures of $\mathcal{C}_{T \rightarrow S}(l)$ so that they take into account the improved knowledge.

- Updating Structure Estimation: when the convergence of a tissue segmentation algorithm is reached, it activates the corresponding structure estimation procedures of $\mathcal{C}_{S \rightarrow T}(c)$ only if tissue intensity models have sufficiently changed. This threshold guarantees that the algorithm stops.

LOCUS-TS then integrates information from both tissue and subcortical structure segmentations. Models are mutually constrained, making them gradually more accurate. Unlike global approaches, local model estimation procedures are asynchronously scheduled by mutual interactions that ensure consistency of the local knowledge.

\section{EVALUATION}

\section{A. Implementation}

The apparent complexity of LOCUS is well reduced by considering its implementation in a multi-agent framework. Following the approach of [32] we implemented our local and cooperative approach (see Figure 7) in a simulated memoryshared multi-agent system inspired by MadKit ${ }^{1}$. We defined one agent, i.e. an autonomous process, per subvolume. Such a multi-agent framework is a convenient way to implement local computation, cooperation and coordination mechanisms between autonomous processes (for architecture details see [40]). In our model, all local EM procedures share the same grey level, labeling and parameter maps. Each local estimation procedure modifies only the labeling map for its dedicated subvolume, but has access to neighboring voxels in different subvolumes for the computation of the spatial correlation term. Then, the consistency of neighboring voxels in different subvolumes is correctly modeled.

We implemented different algorithms for MRF parameters estimation: the ICM algorithm [37] and the EM-based algorithms such as the mode-field, mean-field and simulated-field algorithms proposed in [38]. We chose not to estimate the $\eta$ parameters that account for spatial interaction in MRF but rather considered it as $\eta=1 / T$ with $\mathrm{T}$ a decreasing temperature as proposed in [37]. In practice, $T$ decreasing from 10 to 5 provided good results. We choose a 3-Dimensional 26-neighborhood system for both voxel and local model neighborhood. For LOCUS-T we experimentally set the size of subvolumes to $20^{3}$ voxels, resulting into 300 to 500 distributed agents. We set $\delta_{\text {keep }}=0.3$ and $\delta_{\text {replace }}=1.0$ for model correction, $M A X_{\text {tissue_estimation_restart }}=5$ to ensure the

\footnotetext{
${ }^{1}$ see http://www.madkit.org
} 
end of LOCUS-T and $\delta_{\mathrm{ROI}}=0.20$ for ROI extraction of structure subvolumes. The method performance does not seem sensitive to variations of most of these parameter values. Parameters $\delta_{\text {keep }}$ and $\delta_{\text {replace }}$ are thresholds relative to the Kullback-Leibler distance between two Gaussians. In all the experiments we performed, we did not observe significant dependency on these parameter values. We kept the same values for various T1-weighted structural MR brain scans and tests performed on T2-weighted and Flair images did not require modifications of these parameters. However, more experimentation would be necessary to really quantify the influence of these parameters with respect to the different MRI modalities. As regards parameters $\eta^{c}$, choosing them as increasing from 0.1 to 0.2 gives good results on both 1.5Tesla and 3Tesla brain scans. The subvolume size is a mildly sensitive parameter. In practice, subvolume size from $20 \times 20 \times 20$ to $30 \times 30 \times 30$ gives similar good results on high resolution images $\left(1 \mathrm{~mm}^{3}\right)$. On low resolution images, a size of $25 \times 25 \times 25$ may be preferred. For LOCUS-TS we considered $L=9$ subcortical structures: the ventricular system, the two frontal horns, the two caudate nuclei, the two thalamus, and the two putamens. The parameter $\gamma_{i}$ controls the influence of the external field for structure segmentation. We observed that its value could affect more significantly the segmentation results. In practice, we obtained good results by introducing the entropy of the posterior probabilities in the expression of $\gamma_{i}$. The $\gamma_{i}$ influence is choosen to be larger when two tissue probabilities are almost equal, to prevent instability in the labelling. This introduces contrast-dependent knowledge, reinforcing robustness to various images. Experimentally, $\gamma_{i}=0.75+H_{i}$ gives good results.

Our tool was developed in a very modular programming way which allows us to extend it easily, for instance to introduce new anatomical relations for the segmentation of new structures. A graphical interface of the system enables us to observe in real time the evolution of each agent distributed all over the volume. It records and displays several steps, such as model estimation, model correction and agent coordination. It also gives the possibility to observe a large amount of information during the computation: the intensity based probabilities, the MRF regularized probabilities, the number of DILEM iterations for each subvolume, the interpolated values of intensity model means and variances, the fuzzy maps of each spatial relation, the merged fuzzy maps for each structure and a 3-Dimensional reconstruction of structures (See [40] for more information). All evaluation experimentations were performed on a Pentium4 3Ghz with 2GB RAM.

\section{B. Data}

The evaluation was performed using both phantoms and real $3 \mathrm{~T}$ brain scans. We used the normal $1 \mathrm{~mm}^{3}$ BrainWeb phantoms database from the McConnell Brain Imaging Center [41]. These phantoms are generated from a realistic brain anatomical model and a MRI simulator that simulates MR acquisition physics, in which different values of nonuniformity and noise can be added. Because these images are simulated

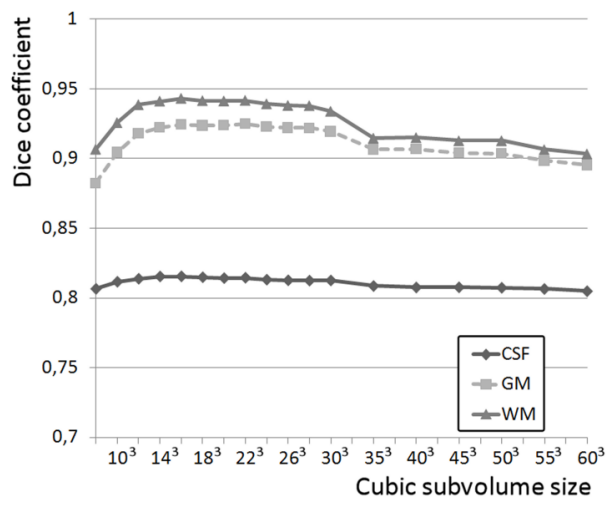

Fig. 8. Evaluation of the sensitivy of LOCUS-T to the subvolume size on the $5 \%$ noise, $40 \%$ inhomogeneity BrainWeb phantom. This image shows the Dice coefficient obtained for the three tissues for different subvolume size from $8 \times 8 \times 8$ to $60 \times 60 \times 60$.

we can quantitatively compare our tissue segmentation to the underlying tissue generative model to evaluate the segmentation performance. As in [13], [15], [16] we performed a quantitative evaluation using the Dice similarity metric [42]. This metric measures the overlap between a segmentation result and the gold standard. By denoting by $\mathrm{TP}_{k}$ the number of true positives for class $k, \mathrm{FP}_{k}$ the number of false positives and $\mathrm{FN}_{k}$ the number of false negatives the Dice metric is given by:

$$
d_{k}=\frac{2 \mathrm{TP}_{k}}{2 \mathrm{TP}_{k}+\mathrm{FN}_{k}+\mathrm{FP}_{k}}
$$

and $d_{k}$ takes its value in $[0,1]$ where 1 represents the perfect agreement. Since BrainWeb phantoms contain only tissue information, three subcortical structures were manually segmented by three experts: the left caudate nucleus, the left putamen and the left thalamus. We then computed our structure gold standard using STAPLE [43], which computes a probabilistic estimate of a true segmentation from a set of different manual segmentations. We also evaluated LOCUS-T on two datasets coming from the Internet Brain Segmentation Repository (IBSR) of Harvard Laboratory ${ }^{2}$. We used the 20 normal T1-weighted brain scans, which have lower resolution and lower contrast than BrainWeb phantoms. They contain some acquisition related artifacts that make classification difficult. We also used the recent $181 \times 1 \times 1.5 \mathrm{~mm}$ IBSR scans, which are higher quality images. Finally, we evaluated LOCUS-T and LOCUS-TS on real 3T MR brain scan (T1 weighted sequence, $\mathrm{TR} / \mathrm{TE} /$ Flip $=12 \mathrm{~ms} / 4.6 \mathrm{~ms} / 8^{\circ}$, Recovery Time $=2500 \mathrm{~ms}$, Acquisition Matrix $=256 \times 256 \times 176$, voxel isotropic resolution $1 \mathrm{~mm}^{3}$ ) coming from the Grenoble Institut of Neuroscience (GIN).

\section{Evaluation of LOCUS-T}

We first evaluated with BrainWeb phantoms the contribution of the different algorithms for the estimation of MRF parameters: ICM [37] and the three mean-field like algorithms proposed by Celeux et al. [38]. Mode-field algorithm appeared to be

\footnotetext{
${ }^{2}$ http://www.cma.mgh.harvard.edu/ibsr/
} 

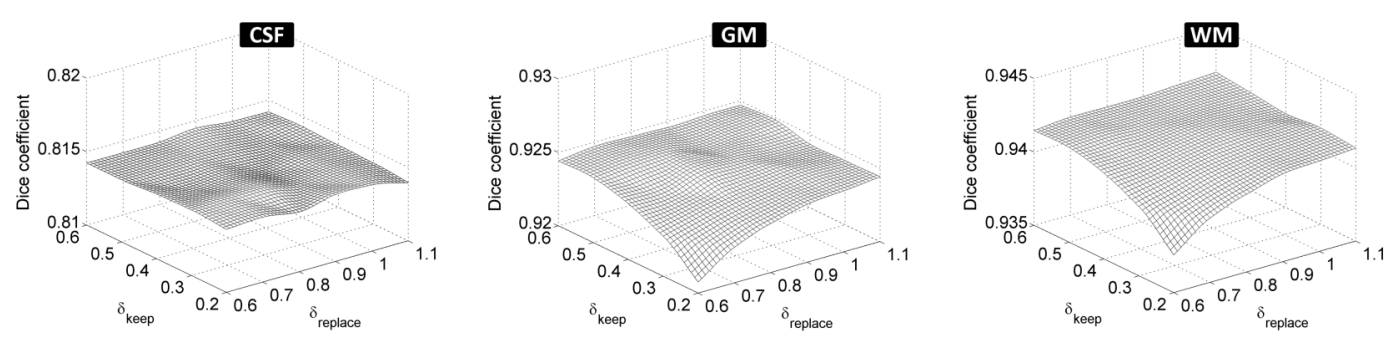

Fig. 9. Evaluation of the sensitivy of LOCUS-T to the model checking and model corrections parameters on the $5 \%$ noise, $40 \%$ inhomogeneity BrainWeb phantom. The three images shows for the three tissues the Dice coefficient obtained for different values of $\delta_{\text {keep }}$ and $\delta_{\text {replace }}$.

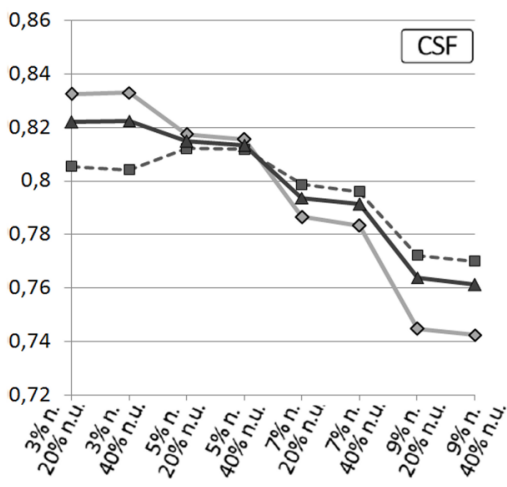

(a)

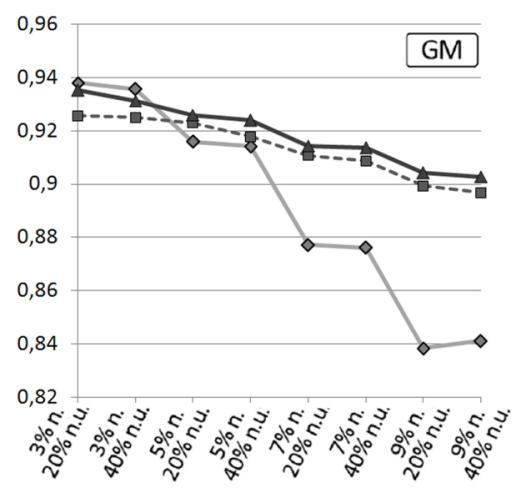

(b)

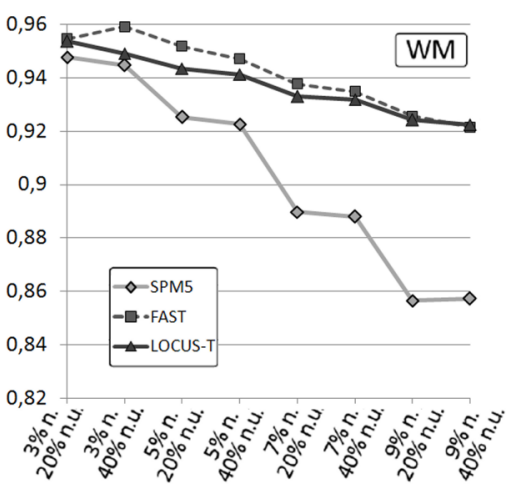

(c)

Fig. 10. Evaluation of LOCUS-T and comparison to SPM5 and FAST on eight BrainWeb phantoms, for different values of noise and nonuniformity. For example "3\% n., $20 \%$ n.u." means "Phantom with $3 \%$ of noise and $20 \%$ of nonuniformity". The corresponding Dice values are shown for CSF (a), GM (b) and WM (c).

approximately equivalent in time and performances to ICM. For T1-weighted MR brain scans, we did not find better results using the mean-field and simulated-field algorithms, which increase the estimation computational cost. This is maybe due to the low contrast between tissues. We therefore chose the mode-field algorithm for the estimation of MRF parameters in LOCUS.

We then evaluated the sensitivity of LOCUS-T to the subvolume size parameter. Figure 8 shows the Dice coefficient obtained for different subvolume sizes on the 5\% noise, $40 \%$ inhomogeneity BrainWeb phantom. Such an inhomogeneity level can be considered as the maximum level obtained with modern scanners. We also evaluated the sensitivity of the approach to model checking and model correction parameters on the same BrainWeb phantom (see Figure 9).

We then quantitatively compared LOCUS-T to two well known tissue segmentation tools, FAST [4] from FSL and SPM5 [13]. Note that LOCUS-T and FAST require a skull-stripping step before the segmentation whereas for SPM5 brain extraction is embedded into the segmentation procedure. To avoid the introduction of errors due to skull-stripping operation, we used a common brain mask for all the tested methods. We first segmented BrainWeb phantoms with SPM5 and used the brain mask obtained as the input for LOCUS-T and FAST. Figure 10 shows the evaluation performed on eight BrainWeb phantoms, for $3 \%, 5 \%, 7 \%$ and $9 \%$ of noise with $20 \%$ and $40 \%$ of nonuniformity for each noise level. The mean Dice metric over all experiments and for all tissues is $86.4 \%$ for SPM5, $88.4 \%$ for FAST and $88.5 \%$ for LOCUS-T. The mean computation times for the full 3-D segmentation were $4 \mathrm{~min}$ for LOCUS-T, 8min for FAST and more than 10min for SPM5. We then evaluated the tissue segmentation performance on the 20 normal T1-weighted brain scans from IBSR (see Figure 11(a)). We compared it to other published results by transforming the publicly available Tanimoto coefficients $t_{k}$ of different approaches ${ }^{3}$ to Dice coefficients $d_{k}$ via the formula: $d_{k}=2 t_{k} /\left(t_{k}+1\right)$ (see [16]). We observed that with these low resolution, low contrast and highly artifacted images LOCUS-T and FAST are penalized compared to SPM5. The use of MRF models in these two approaches appears to over-regularize the labeling in such low-resolution conditions, whereas SPM5 provides better results. On the 18 IBSR 1x1x1.5mm scans (see Figure 11(b)) SPM5 appears slightly better for CSF and GM but less efficient for WM.

The Figure 12 shows a visual evaluation on a very high bias field brain scan. This image (1.5T T1-weighted, TR/TE/Flip $=24 \mathrm{~ms} / 6 \mathrm{~ms} / 30^{\circ}, \quad \mathrm{FOV}=192 \times 192 \mathrm{~mm}^{2}$, voxel resolution $=0.75 \times 0.75 \times 1 \mathrm{~mm}$ ) was acquired with a surface coil placed on the occipital part of the head which provides a high sensitivity in a small region (here the occipital lobe). Such an acquisition is useful for functional imaging but results in highly nonuniform intensities. SPM5, which uses an $a$

\footnotetext{
${ }^{3}$ See http://www.cma.mgh.harvard.edu/ibsr/result_jcr_0997.html
} 


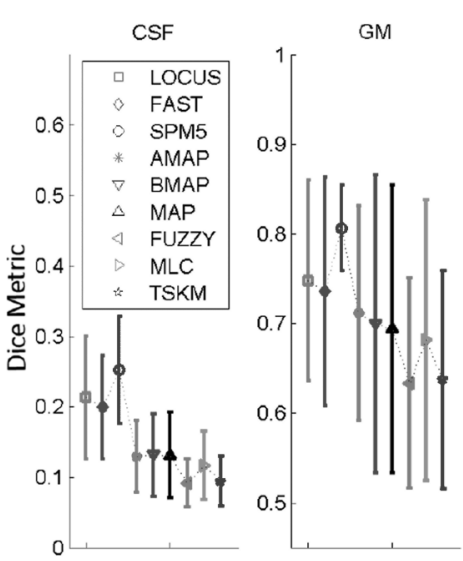

(a)
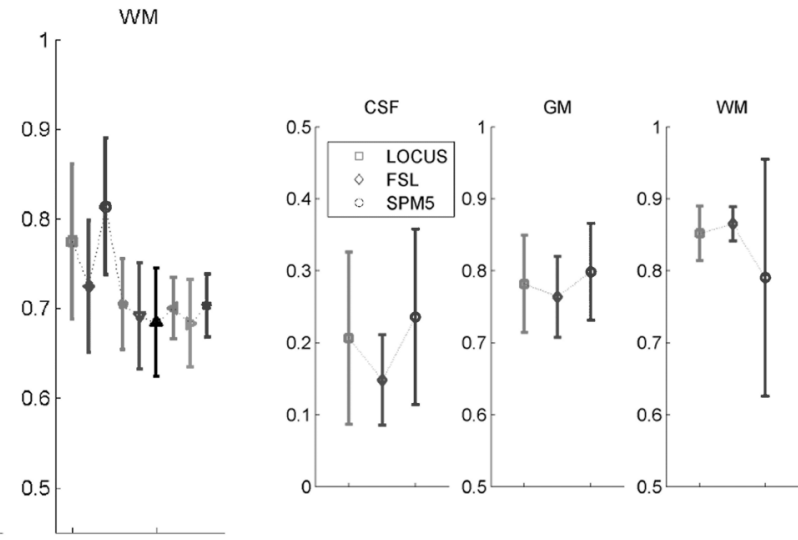

(b)

Fig. 11. Evaluation of LOCUS-T on the two IBSR datasets. Image (a) shows the average Dice metric and its variance on the 20 brain scans from IBSR for each tissue class for LOCUS-T, FAST and SPM5 and other published methods (Maximum Aposteriori Probability, Adaptive-MAP, Biased-MAP, Fuzzy C-Means, Maximum Likelihood, Tree-Structure K-Mean). Image (b) shows the evaluation on the 18 1x1x1.5mm IBSR scans.

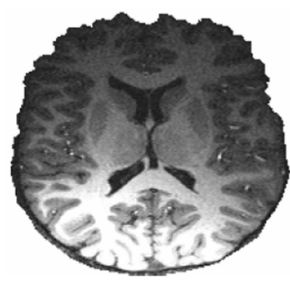

(a)

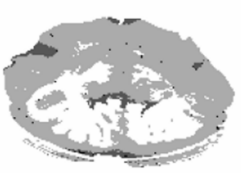

(b)

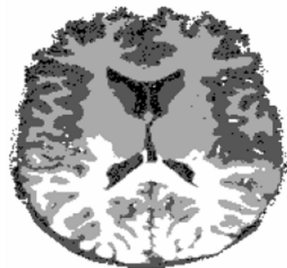

(c)

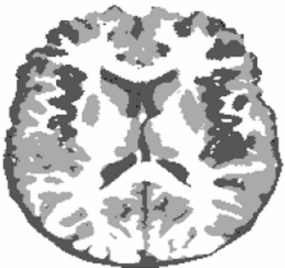

(d)

Fig. 12. Evaluation on a MR brain scan acquired with a surface coil (a), resulting in very high intensity nonunformities. Tissue segmentation provided by SPM5 (b), FAST (c) and LOCUS-T (d).

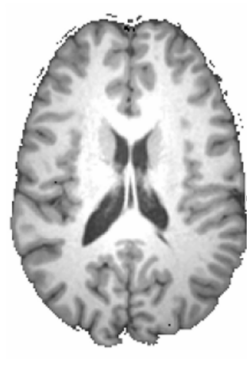

(a)

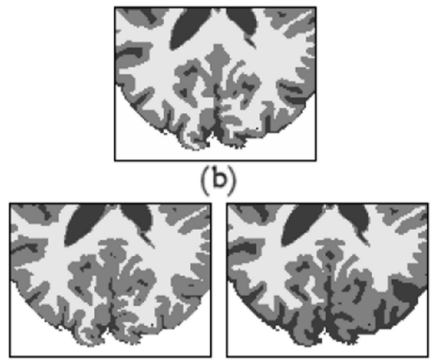

(c)

(d)
Fig. 13. Visual comparison of the robustness to nonuniformity on a real $3 \mathrm{~T}$ brain scan (a). Image (b) is the segmentation provided by LOCUS-T, (c) by SPM5 and (d) by FAST.

priori atlas, failed in the segmentation probably because of the difficulty to match the atlas with such a surface coil brain acquisition. FAST did not estimate a correct bias field. Our local approach clearly appears to be more robust to very high intensity nonuniformities.

The Figure 13 shows the tissue segmentation results on a real high resolution $3 \mathrm{~T}$ brain scan. LOCUS-T and SPM5 appear as more robust to intensity nonuniformity than the FAST bias field correction.

Finally, we observed for different positions in the brain the proportion of local MRF models that require various numbers of DILEM iterations. The Figure 14 shows the average proportions over 8 BrainWeb image segmentations for different values of noise $(3 \%, 5 \%, 7 \%$ and $9 \%)$ and nonuniformity $(20 \%$ and $40 \%$ ).

\section{Evaluation of the cooperative tissue and structure segmen- tation - LOCUS-TS}

We next evaluated the cooperative tissue and structure segmentation LOCUS-TS. The Figure 15(a) shows the evaluation of the structure segmentation on eight BrainWeb phantoms with different value of noise and nonuniformity. This evaluation was realized by computing the Dice similarity metric between the structure segmentation provided by LOCUS-TS and our structure gold standard computed by STAPLE. The mean Dice metric over all experiments is $82.7 \%$ for the caudate nucleus, $73.9 \%$ for the putamen and $71.6 \%$ for the thalamus. It shows the caudate nucleus segmentation to be more robust to noise and to nonuniformity than for the two other structures. This is due to a higher contrast between GM and WM within this structure. We compared our approach to the structure segmentation performed by Freesurfer [20] on the 5\% of noise and $40 \%$ of nonuniformity image. The Dice metric values were respectively $88 \%, 86 \%$ and $90 \%$ for the caudate nucleus, the putamen and the thalamus, which is better than the values 


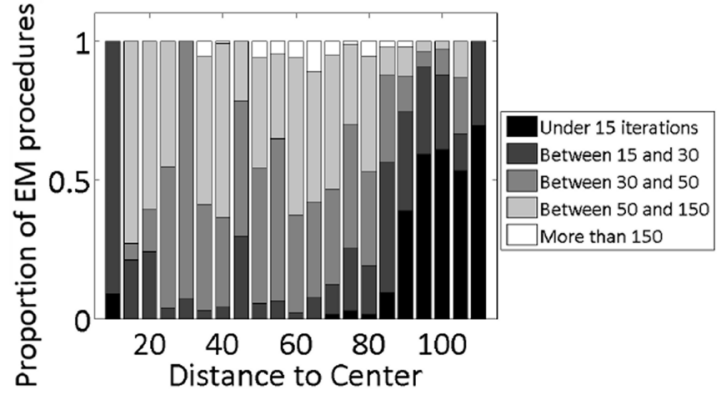

(a)

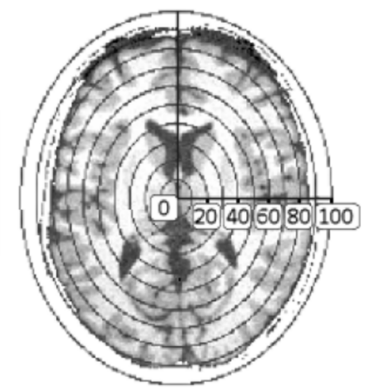

(b)

Fig. 14. Image (a) shows the average proportions, over eight BrainWeb images LOCUS-T segmentations, of local EM procedures that require various number of DILEM iterations to converge. Theses proportions are computed for different positions in the brain (see Image (b)). The position of a MRF $M_{c}^{T}$ is defined by an elliptic distance between the brain center and the subvolume $V_{c}^{T}$ center.

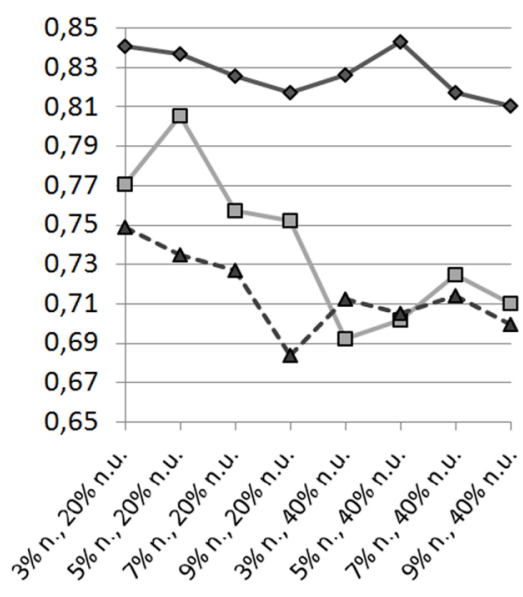

(a)

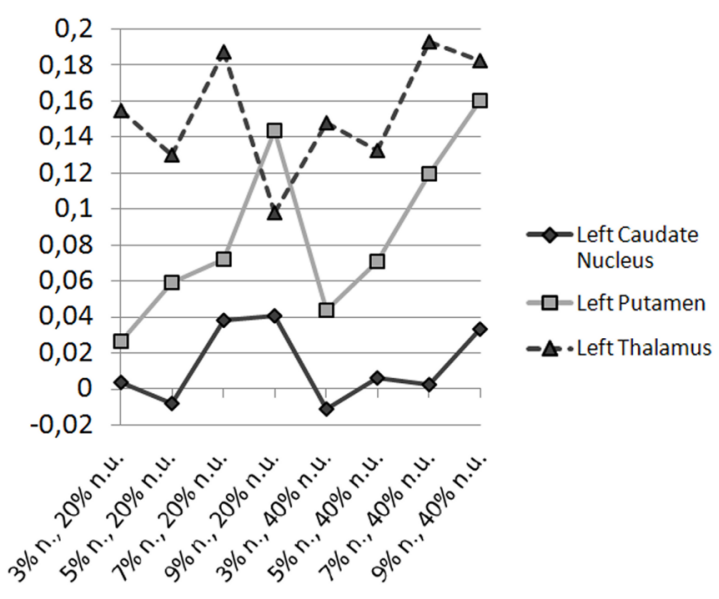

(b)

Fig. 15. Image (a) shows the evaluation of the structure segmentation performed on eight images from the BrainWeb database with different noise and nonuniformity values. Image (b) shows the relative improvement of the Dice similarity metric between the first and the last convergence for each structure.

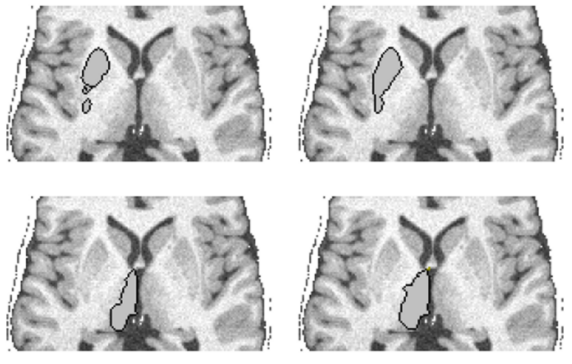

Fig. 16. Qualitative relative improvement of the putamen segmentation (first row) and thalamus segmentation (second row) between the first convergence (column 1) and the last (column 2) convergence, on the BrainWeb phantom with $5 \%$ of noise and $40 \%$ of nonuniformity.

obtained using LOCUS-TS (respectively $84 \%, 70 \%$ and $71 \%$ ). The computation time was however more than 15 hours for all the 37 structures, while LOCUS-TS ran in 15 minutes.

The benefits of the feedback on tissue segmentation could not be evaluated properly because putamens and thalamus appear to be under segmented on the BrainWeb tissue gold standard.
Rather, we computed the relative improvement of the Dice metric value between the first and the last convergence for each structure (see Figure 15(b)). Denoting by $d_{s}^{(0)}$ the value of the Dice metric at the first convergence and by $d_{s}^{(f)}$ the value at the last convergence, we computed it by $\frac{\left|d_{s}^{(0)}-d_{s}^{(f)}\right|}{d_{s}^{(0)}}$. It gives an indication of the benefits of combining tissue and structure segmentation. We observed only a small impact for the caudate nucleus $(+1 \%$ in average), likely because its GM radiometry is well contrasted with WM radiometry, providing a good segmentation at the first convergence. We observed a clear benefit for the thalamus ( $+15 \%$ in average) and for the putamen (+9\% in average). In Figure 15(b) we also remark the improvement of putamen segmentation to be more important with noisy images. The Figure 16 illustrates the putamen and thalamus improvements on the 5\% noise, $40 \%$ nonuniformity BrainWeb phantom.

The Figure 17 shows a qualitative evaluation of LOCUS-TS on three different real $3 \mathrm{~T}$ brain scans. It visually clearly shows the benefits provided by LOCUS-TS compared to LOCUS-T, showing improved tissue segmentation in the regions of the 


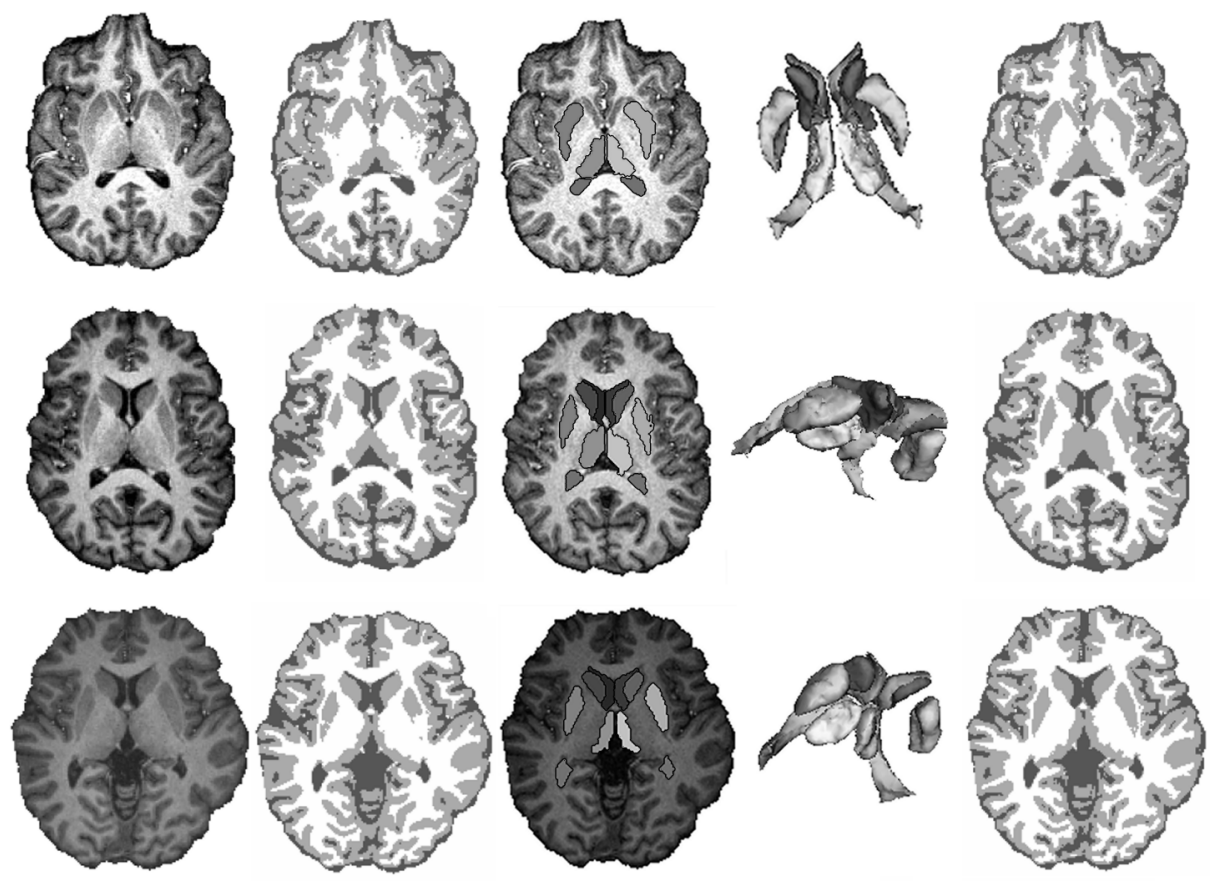

Fig. 17. Evaluation of cooperative tissue and structure segmentation on three real 3T brain scans shown in the first column. The corresponding tissue segmentations provided by LOCUS-T are shown in the second column. The third and fourth columns show respectively the 3-Dimensional reconstructions of subcortical structures provided by LOCUS-TS and the corresponding tissue segmentations.

putamen and the thalamus.

Finally, we performed an image simulation. The idea was to consider different segmentation models and to simulate the intensity of each voxel from the parameters of each model. If we denote by $\bar{y}_{i}$ the simulated intensity of voxel $i$ and by $\bar{G}_{\mu, \sigma}$ one random Gaussian sample of mean $\mu$ and variance $\sigma$, we performed simulation by:

$$
\overline{y_{i}}=\sum_{k=1 . . K} p\left(t_{i}=e_{k} \mid y_{i}, \Phi\right) \bar{G}_{\mu_{i, k}, \sigma_{i, k}}
$$

The Figure 18 shows the different simulation results with three specific areas annotated on Figure 18(a). We first simulated an image from the parameters of a single global MRF model. In the annotated areas the simulation is not accurate because a global intensity model without any bias field modelization does not take into account intensity nonuniformity (see Figure $18(\mathrm{~b})$ ). We then simulated an image from LOCUS-T parameters (see Figure 18(c)). In the areas 1 and 2 the simulation is more accurate since LOCUS-T intrinsically models the nonuniformity by estimating local tissue intensity models. In the area 3 , the simulation of the thalamus and the putamen is improved with LOCUS-T but not satisfyingly. We also observe that the bias field in White Matter is better simulated (see intensity of WM from bottom to top). With LOCUS-TS (see Figure 18(d)) nonuniformity due to tissue properties is well modeled and the simulation appears to be more satisfying.

\section{Discussion}

MR brain scan segmentation is a difficult task and requires the introduction of a priori knowledge, such as bias field models or anatomical atlases. Our approach minimizes a priori knowledge insertion and time computation by considering (1) a local approach to cope with intensity nonuniformity, (2) dynamically updated fuzzy anatomical relations to deal with structure segmentation and (3) a multi-agent based implementation for distributed problem solving. In addition, we introduced natural dependencies for model regularization. LOCUS compliments the local MRF voxel regularization with (1) regularization between local subvolumes, and (2) regularization between tissue and structure segmentation.

Unlike global approaches, our LOCUS-T approach does not require an explicit modelization of the nonuniformity artifact, generally modeled as a single slowly varying bias field affecting all tissue classes equally. LOCUS-T takes advantage of local models estimation to accurately reflect the local intensity distributions. It is thus able to handle multiple sources of nonuniformity, including nonuniformities due to hardware imperfections and nonuniformities due to tissue biological properties. However, despite their ability to perform local estimation over subvolumes, local approaches face some difficulties. First, classes are likely to be under-represented in some subvolumes, leading to an unreliable estimation. Second, the locality makes the approach more sensitive to noise. In consequence, most local approaches to date use overlapping subvolumes to estimate local intensity models with a sufficient number of voxels. This appears to be efficient (but not robust to noise) when the local estimation is performed by a Fuzzy CMean algorithm [18], which is not too computationally costly. However, when introducing MRF modelization for a robustto-noise segmentation, such a local overlapping approach has an extremely high computational cost, like the A-MAP algorithm of Rajapakse et al. [17]. Consequently we propose a local approach with (1) MRF models for a robust-to-noise 


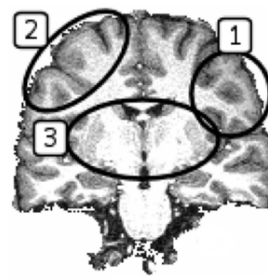

(a)

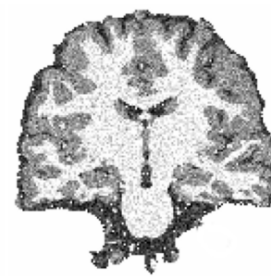

(b)

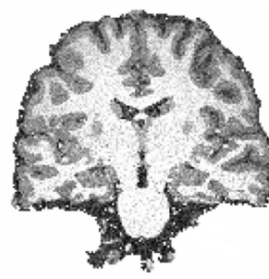

(c)

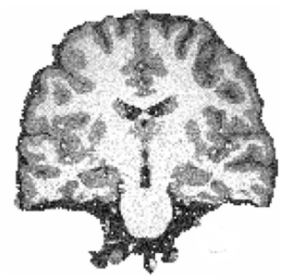

(d)

Fig. 18. Image simulation performed from the parameters of the segmentation model, with a gradually more complex segmentation model. (a) is the original image, (b) a simulation from the parameters of a global MRF segmentation model, (c) a simulation from LOCUS-T parameters and (d) a simulation from LOCUS-TS parameters. The marks 1,2,3 indicate areas showing a clear improvement linked to the model refinement.

segmentation, (2) non-overlapping subvolumes for computational time considerations and (3) local cooperation between neighboring models to ensure the global consistency of local models in an elegant and efficient way. We use cubic-spline interpolation to compute one intensity model for each voxel from neighboring local estimations. Our approach appears thus to approximate non stationary MRF approaches in a tractable way. For the estimation of MRF parameters we used the modefield algorithm [38], equivalent to the EM algorithm used in [3], [4]. We did not observe significant improvements when using the other mean field-like algorithms proposed in [38]. Our local tissue segmentation approach appears to be robust to model checking and model correction parameters $\delta_{k e e p}$ and $\delta_{\text {replace }}$ that are central to ensure the consistency of local intensity models. CSF segmentation is extremely low sensitive to these parameters, and GM or WM segmentation are low sensitive: we observe only a difference of $0.5 \%$ between the best and the worst dice coefficient obtained. In general, the results are very stables for values of $\delta_{\text {keep }} \geq 0.3$ and values of $\delta_{\text {replace }} \geq 0.8$. We also observe LOCUS-T to be robust to the subvolume size parameter : the choice of the size between $15 \times 15 \times 15$ (i.e. 3375 voxels) and 30x30x30 (i.e. 27000 voxels) gives approximatively the same segmentation result.

The partial volume effect (PVE) is currently not taken into account. The simple addition of extra classes to model partial volume voxels with a Gaussian density may work well with our approach but is likely to require larger subvolumes so that classes are sufficiently represented. It would be more efficient to study more realistic PVE models such as those proposed by Santago et al. [44] or Van Leemput et al. [45].

The LOCUS-T tissue segmentation results are similar to other approaches (SPM5, FAST) on high resolution images with shorter computational time. Note however that SPM5 is more sensitive to noise (see Figure 10). The time gain obtained by LOCUS-T is due to the locality: easy-to-segment subvolumes converge quickly, allowing the system to focus on other areas. Because of complex dependencies between subvolumes it is not easy to infer general behaviors about the number of DILEM iterations required to reach the convergence. However, as seen in Figure 14, it appears that MRF models in the peripheral region of the brain generally require low number of iterations. These subvolumes are likely to contain less brain voxels and under-represented classes. The model correction process will then often replace the local model by the neigh- boring mean model. This later model generally appears to be consistent with the voxels of the subvolume so that the DILEM loop converges quickly. On the contrary, MRF showing a large number of DILEM iterations are mainly localized between $40 \mathrm{~mm}$ and $80 \mathrm{~mm}$. This is probably due to the presence of a lot of partial volume voxels which makes the cortex grey matter difficult to segment in this low contrast region. We also notice that subvolumes in which the three tissues are well represented generally require more iterations to reach the stabilization and get a fine estimation of the three tissue models. These experiments demonstrate the flexibility of our approach, which adapts to the local image complexity by spending locally more time when needed and grounding model accuracy on the most informational zones. The coordination mechanisms provide an asynchronous scheduling of estimation procedure execution which ensures the information diffusion between models.

The evaluation on very high intensity nonuniformity images acquired with a surface coil (Figure 12) points out the limitations of using too rigid prior knowledge in a segmentation model. It shows (1) the limitations of atlas registration (SPM5), atlas being hard to match on such specific images, and (2) the limitations of the FAST underlying bias field modelization. LOCUS-T, whose only nonuniformity assumption is that intensities vary slowly over the volume, appears to be more robust to such very high nonuniformity. On the low resolution and low contrast images from the IBSR database (see Figure 11), LOCUS-T and the FAST algorithm of FSL are less efficient, which is probably due to an over regularization of the MRFs on such images.

For structure segmentation we bypass the use of an atlas to provide an a priori knowledge [20], [35]. These methods are expected to segment a higher number of structures but atlas registration algorithms are extremely time consuming. Their performances are very sensitive to the atlas precision and to registration errors that generally appear in low contrast regions. Such approaches are perturbed by inter-individual variability or pathological cases that make the image too distant from the atlas. Shape Modeling [21] appears as an interesting approach but requires an important learning step that may not handle pathological MRI as well. Contrary to these approaches introducing a strong a priori, we favor a more general knowledge: we describe the brain anatomy via a set of stable fuzzy spatial relations between structures. With this framework we compute a fuzzy area where the structure is likely to be present, which 
is less restrictive than probabilistic atlases. In the approach of [23] such knowledge was introduced in a deformable model to segment seven subcortical structures on 1.5T MR brain scans. The efficiency of deformable models however critically relies on fundamental data preprocessing steps to stabilize the deformable models on the true frontiers of the objects. This approach is then sensitive to noise in low-contrast regions. In [23] no results were reported on the putamen which is a highly nonuniform bright grey structure, and on high field images (3T or higher). With a similar approach, Atif et al. [46] reports segmentation results on subcortical structures in presence of a tumor, showing that some relations are stable even for pathological brain scans. Note that if the reference structure is located incorrectly, the prior knowledge drawn could be harmful. However, our hierarchical construction of fuzzy spatial relations maps with an iterative updating of the spatial relation knowledge, limits the impact of such a mislocation. In practice, the well-defined contrast property of the ventricular system makes it easier to segment. Then, other structures can be progressively segmented. Additionally, in our approach, the fuzzy spatial relations knowledge is not static but is updated and improved during iterations. We introduce the fuzzy localization constraints in a MRF framework. Fischl et al. [20] introduced the knowledge of a prior registered atlas via the interaction energy term of a global MRF. This model is however simplified for tractability. They considered that the dependence of a label on its neighbors can be expressed as the product of the probabilities given each of the neighbors separately. This simplifying assumption reduces the Markov property. Here we consider a different way to introduce $a$ priori knowledge in a MRF. We extend the traditionally used Potts Model with an external field parameter that allows us to introduce the fuzzy localization constraints as relative prior weights for each voxel. Our approach labels nine structures, including the putamen. The results are not as good as those obtained with an atlas based approach such as Freesurfer [20]. However its computational efficiency can be an advantage for some clinical applications. Fuzzy description of brain anatomy is then a promising way to introduce a priori knowledge at a low computational cost and should be considered and improved in the future to yield fast and reliable structure segmentation tools.

In addition we propose to link tissue and structure segmentations so that they mutually improve. The benefits are mainly visible for putamen or thalamus. Indeed these structures present a higher myelin fiber density than the caudate nucleus and appear in a brighter grey intensity on T1-weighted MR brain scans. The feedback of structure segmentation on tissue segmentation provides valuable additional knowledge to locally correct tissue intensity models.

Note that the presence of a pathology such as a stroke lesion, a tumor or multiple sclerosis lesions is likely to highly perturb the local estimation of tissues models. If we segment such images using 4 classes (CSF, GM, WM and Pathology), we observe that cooperation and coordination mechanisms are not sufficient: the modelization of a class corresponding to a small portion of the volume fails to be spread over all the volume. To extend our LOCUS-T approach to these pathological cases, one solution would be to assess the right number of classes in each subvolume. This could be done by a model selection criterion such as the extension of the Bayesian Information Criterion (BIC) proposed in [47]. Each distributed MRF would then estimate local tissue intensity models with a variable number of classes. This would require the definition of a specific model checking step that is able to handle possibly different number of classes between neighboring subvolumes. Besides, an adaptive partitioning of the volume could be an interesting refinement for LOCUS-T. It could yield to different sizes and shapes for the subvolumes depending on the brain regions, making the local estimation more accurate. Finally, we currently use a simulated distributed system to evaluate our LOCUS approach. A true distributed version may lead to extremely low computation time and could be of great interest for clinical use.

This original combination of local distributed Markov models, which includes anatomical knowledge, then appears to be a powerful and promising approach to reliable and efficient MR brain scan segmentation.

\section{APPENDIX A \\ BACKGROUND CLASS INTENSITY MODEL FOR STRUCTURE SEGMENTATION.}

We propose to compute $p\left(y_{i} \mid s_{i}^{l}=e_{B}, \Psi_{y}^{l}\right)$ as follows. We have,

$$
\begin{aligned}
& p\left(y_{i} \mid s_{i}^{l}=e_{B}, \Psi_{y}^{l}\right)=p\left(y_{i} \mid s_{i} \neq e_{l}, \Psi_{y}^{l}\right) \\
& =\sum_{k=1}^{3} p\left(y_{i} \mid t_{i}=e_{k}, s_{i} \neq e_{l}, \Psi_{y}^{l}\right) p\left(t_{i}=e_{k} \mid s_{i} \neq e_{l}, \Psi_{y}^{l}\right) .
\end{aligned}
$$

We consider that $p\left(y_{i} \mid t_{i}=e_{k}, s_{i} \neq e_{l}, \Psi_{y}^{l}\right)=p\left(y_{i} \mid t_{i}=e_{k}, \Phi_{y}\right)$ and that a voxel of tissue class $e_{k}$ not belonging to the structure $e_{l}$ is either a voxel of an other structure of tissue $e_{k}$ or a voxel of the background so that, omitting the parameters in the notation,

$$
\begin{aligned}
& p\left(t_{i}=e_{k} \mid s_{i} \neq e_{l}\right)= \\
& \sum_{l^{\prime} \neq l, T^{l^{\prime}}=e_{k}} p\left(t_{i}=e_{k} \mid s_{i}=e_{l^{\prime}}\right) \pi_{l^{\prime}, \bar{l}}+p\left(t_{i}=e_{k} \mid s_{i}=e_{0}\right) \pi_{B, \bar{l}} \\
& =\sum_{l^{\prime} \neq l, T^{l^{\prime}}=e_{k}} \pi_{l^{\prime}, \bar{l}}+p\left(t_{i}=e_{k} \mid s_{i}=e_{0}\right) \pi_{B, \bar{l}}
\end{aligned}
$$

with:

$$
\left\{\begin{array}{l}
\pi_{l^{\prime}, \bar{l}}=p\left(s_{i}=e_{l^{\prime}} \mid s_{i} \neq e_{l}\right)=\frac{p\left(s_{i}=e_{l^{\prime}}\right)}{p\left(s_{i} \neq e_{l}\right)}, \\
\pi_{B, \bar{l}}=p\left(s_{i}=e_{0} \mid s_{i} \neq e_{l}\right)=\frac{p\left(s_{i}=e_{0}\right)}{p\left(s_{i} \neq e_{l}\right)} .
\end{array}\right.
$$

We assume that $p\left(s_{i}=e_{l^{\prime}}\right)$ is given by the fuzzy localization map of structure $l^{\prime}$, ie. $p\left(s_{i}=e_{l^{\prime}}\right) \propto f_{i}^{l^{\prime}}$ and that $p\left(s_{i}=e_{B}\right) \propto f_{i}^{B}$ where $f_{i}^{B}$ is given by $f_{i}^{B}=1-\max _{l} f_{i}^{l}$. It follows that,

$$
\begin{array}{r}
\pi_{l^{\prime}, \bar{l}}=f_{i}^{l^{\prime}} / D, \quad \pi_{B, \bar{l}}=f_{i}^{B} / D \\
\text { with } D=\sum_{k \neq l} f_{i}^{k}+f_{i}^{B} .
\end{array}
$$

Assuming in addition that $p\left(t_{i}=e_{k} \mid s_{i}=e_{0}\right)=p\left(t_{i}=e_{k}\right)$, it comes,

$$
\begin{aligned}
p\left(y_{i} \mid s_{i} \neq e_{l}, \Psi_{y}^{l}\right)= & \sum_{k=1}^{3} p\left(y_{i} \mid t_{i}=e_{k}, \Phi_{y}\right) \\
& {\left[\sum_{l \neq l^{\prime}, T^{l^{\prime}}=e_{k}} \pi_{l^{\prime}, \bar{l}}+p^{M F}\left(t_{i}=e_{k}, \Phi\right) \pi_{B, \bar{l}}\right], }
\end{aligned}
$$

with $p^{M F}\left(t_{i}=e_{k}, \Phi\right)$ denoting the mean field approximation of the intractable $p\left(t_{i}=e_{k}, \Phi\right)$. 


\section{REFERENCES}

[1] S. Geman and D. Geman, "Stochastic relaxation, Gibbs distributions and the Bayesian restoration of images," IEEE Trans. Pattern Anal. Mach. Intell., vol. 6, pp. 721-741, 1984.

[2] K. Held, E. R. Kopps, B. J. Krause, W. M. Wells, R. Kikinis, and H. W. Muller-Gartner, "Markov random field segmentation of brain MR images," IEEE Trans. Med. Imag., vol. 16, no. 6, pp. 878-886, 1997.

[3] K. Van Leemput, F. Maes, D. Vandermeulen, and P. Suetens, "Automated model-based tissue classification of MR images of the brain," IEEE Trans. Med. Imag., vol. 18, no. 10, pp. 897-908, 1999.

[4] Y. Zhang, M. Brady, and S. Smith, "Segmentation of brain MR images through a hidden Markov random field model and the ExpectationMaximisation algorithm,” IEEE Trans. Med. Imag., vol. 20, no. 1, pp. 45-47, 2001.

[5] J. L. Marroquin, B. C. Vemuri, S. Botello, F. Calderon, and A. Fernandez-Bouzas, "An accurate and efficient Bayesian method for automatic segmentation of brain MRI," IEEE Trans. Med. Imag., vol. 21, no. 8, pp. 934-945, 2002

[6] J. L. Marroquin, E. A. Santana, and S. Botello, "Hidden markov measure field models for image segmentation," IEEE Trans. Pattern Anal. Mach. Intell., vol. 25, no. 11, pp. 1380-1387, 2003.

[7] F. Yang and T. Jiang, "Pixon-based image segmentation with Markov random fields," IEEE Trans. Image Process., vol. 12, no. 12, pp. 1552 1559, 2003.

[8] M. Rivera, O. Ocegueda, and J. L. Marroquin, "Entropy-controlled quadratic markov measure field models for efficient image segmentation," IEEE Trans. Image Process., vol. 16, no. 12, pp. 3047-3057, 2007.

[9] W. D. Rooney, G. Johnson, X. Li, E. R. Cohen, S. G. Kim, K. Ugurbil, and C. S. J. Springer, "Magnetic field and tissue dependencies of human brain longitudinal $1 \mathrm{H} 2 \mathrm{O}$ relaxation in vivo," Magnetic Resonance in Medicine, vol. 57, no. 2, pp. 308-318, 2007.

[10] J. P. Wansapura, S. K. Holland, R. S. Dunn, and W. S. J. Ball, "NMR relaxation times in the human brain at 3.0 tesla," Journal of Magnetic Resonance Imaging, vol. 9, no. 4, pp. 531-538, 1999.

[11] S. Cho, D. Jones, W. E. Reddick, R. J. Ogg, and R. G. Steen, "Establishing norms for age-related changes in proton T1 of human brain tissue in vivo," Magnetic Resonance Imaging, vol. 15, no. 10, pp. 1133-1143, 1997.

[12] W. M. Wells, W. E. L. Grimson, R. Kikinis, and F. A. Jolesz, "Adaptative segmentation of MRI data," IEEE Trans. Med. Imag., vol. 15, no. 4, pp. 429-442, 1996

[13] J. Ashburner and K. J. Friston, "Unified Segmentation," NeuroImage, vol. 26, pp. 839-851, 2005

[14] R. Guillemaud and M. Brady, "Estimating the bias field of MR images," IEEE Trans. Med. Imag., vol. 16, no. 3, pp. 238-251, 1997.

[15] K. Van Leemput, F. Maes, D. Vandermeulen, and P. Suetens, "Automated model-based bias field correction in MR images of the brain," IEEE Trans. Med. Imag., vol. 18, no. 10, pp. 885-896, 1999.

[16] D. W. Shattuck, S. R. Sandor-Leahy, K. A. Schaper, D. A. Rottenberg, and R. M. Leahy, "Magnetic resonance image tissue classification using a partial volume model," NeuroImage, vol. 13, no. 5, pp. 856-876, 2001.

[17] J. C. Rajapakse, J. N. Giedd, and J. L. Rapoport, "Statistical approach to segmentation of single-channel cerebral MR images," IEEE Trans. Med. Imag., vol. 16, no. 2, pp. 176-186, 1997.

[18] C. Zhu and T. Jiang, "Multicontextual fuzzy clustering for separation of brain tissues in magnetic resonance images," NeuroImage, vol. 18, no. 3, pp. 685-96, 2003.

[19] K. M. Pohl, J. Fisher, E. Grimson, R. Kikinis, and W. M. Wells, "A Bayesian model for joint segmentation and registration," NeuroImage, vol. 31, no. 1, pp. 228-239, 2006.

[20] B. Fischl and et al, "Whole brain segmentation: automated labeling of neuroanatomical structures in the human brain." Neuron, vol. 33, no. 3, pp. 341-55, 2002.

[21] K. M. Pohl, J. Fisher, M. Shenton, R. W. McCarley, W. E. L. Grimson, R. Kikinis, and W. M. Wells, "Logarithm odds maps for shape representation," in MICCAI, vol. 4191, 2006, pp. 955-963.

[22] S. Waxman, Correlative Neuroanatomy, 24th ed. McGraw-Hill, New York, 2000

[23] O. Colliot, C. O, and I. Bloch, "Integration of fuzzy spatial relations in deformable models - Application to brain MRI segmentation," Pattern Recognition, vol. 39, no. 8, pp. 1401-1414, 2006

[24] V. Barra and J. Y. Boire, "Automatic segmentation of subcortical brain structures in MR images using information fusion," IEEE Trans. Med. Imag., vol. 20, no. 7, pp. 549-558, 2001.
[25] A. P. Dempster, N. M. Laird, and D. B. Rubin, "Maximum likelihood from incomplete data via EM algorithm," Journal of the Royal Statistical Society, vol. 39, pp. 1-38, 1977.

[26] D. A. Wicks, J. G. Barker, and P. S. Tofts, "Correction of intensity nonuniformity in mr images of any orientation," Magnetic Resonance Imaging, vol. 11, pp. 183-196, 1993.

[27] O. Noterdaeme and M. Brady, "A fast method for computing and correcting intensity inhomogeneities in mri," in Proceedings of the 2008 IEEE International Symposium on Biomedical Imaging: From Nano to Macro (ISBI'08). IEEE, 2008, pp. 1525-1528.

[28] J. G. Sled, A. P. Zujdenbos, and A. Evans, "A non parametric method for automatic correction of nonuniformity in MRI data," IEEE Trans. Med. Imag., vol. 17, no. 1, pp. 87-97, 1998.

[29] J. F. Mangin, "Entropy minimization for automatic correction of intensity nonuniformity," MMBIA, pp. 162-169, 2000.

[30] M. Styner, C. Brechbuhler, G. Szckely, and G. Gerig, "Parametric estimate of intensity inhomogeneities applied to MRI," IEEE Trans. Med. Imag., vol. 19, no. 3, pp. 153-165, 2000.

[31] T. J. Grabowski, R. J. Frank, N. R. Szumski, C. K. Brown, and H. Damasio (2000), "Validation of partial tissue segmentation of singlechannel magnetic resonance images of the brain," NeuroImage, vol. 12, no. 6, pp. 640-656, 2000 .

[32] N. Richard, M. Dojat, and C. Garbay, "Distributed markovian segmentation: Application to MR brain scans," Pattern Recognition, vol. 40, no. 12 , pp. 3467-3480, 2007.

[33] P. A. Yushkevich, J. Piven, H. Cody Hazlett, R. Gimpel Smith, S. Ho, J. C. Gee, and G. Gerig, "User-guided 3D active contour segmentation of anatomical structures: Significantly improved efficiency and reliability," NeuroImage, vol. 31, no. 3, pp. 1116-1128, 2006.

[34] S. C. L. Deonia, B. K. Rutt, A. G. Parrent, and T. M. Peters, "Segmentation of thalamic nuclei using a modified k-means clustering algorithm and high-resolution quantitative magnetic resonance imaging at $1.5 \mathrm{~T}$," NeuroImage, vol. 34, no. 1, pp. 117-126, 2006.

[35] S. Gouttard, M. Styner, S. Joshi, R. G. Smith, H. Cody Hazlett, and G. Gerig, "Subcortical structure segmentation using probabilistic atlas priors," in Medical Imaging 2007: Image Processing., ser. the Society of Photo-Optical Instrumentation Engineers (SPIE) Conference, vol. 6512, 2007, pp. 37-46.

[36] I. Bloch, "Fuzzy relative position between objects in image processing: a morphological approach," IEEE Trans. Pattern Anal. Mach. Intell., vol. 21, no. 7, pp. 657-664, 1999.

[37] Besag, "On the statistical analysis of dirty pictures," Journal of the Royal Statistical Society, vol. 48, pp. 259-302, 1986.

[38] G. Celeux, F. Forbes, and N. Peyrard, "EM procedures using mean field-like approximations for model-based image segmentation," Pattern Recognition, vol. 36, no. 1, pp. 131-144, 2003.

[39] G. Matheron, "Principles of geostatistics," Economic Geology, vol. 58, no. 8, pp. 1246-1266, 1963.

[40] B. Scherrer, M. Dojat, F. Forbes, and C. Garbay, "Agentification of Markov model based segmentation: Application to MRI brain scans," Artificial Intelligence in Medicine, p. to appear, 2008.

[41] D. L. Collins, A. P. Zijdenbos, V. Kollokian, J. G. Sled, N. J. Kabani, C. J. Holmes, and A. C. Evans, "Design and construction of a realistic digital brain phantom," IEEE Trans. Med. Imag., vol. 17, no. 3, pp. 463-468, 1998

[42] L. R. Dice, "Measures of the amount of ecologic association between species," Ecology, vol. 26, pp. 297-302, 1945.

[43] S. K. Warfield, K. H. Zou, and W. M. Wells, "Simultaneous truth and performance level estimation (STAPLE): An algorithm for the validation of image segmentation," IEEE Trans. Med. Imag., vol. 23, no. 7, pp. 903-921, 2004

[44] P. Santago and H. D. Gage, "Quantification of MR brain images by mixture density and partial volume modeling," IEEE Trans. Med. Imag., vol. 12 , no. 3, pp. 566-574, 1993.

[45] K. Van Leemput, F. Maes, D. Vandermeulen, and P. Suetens, "A unifying framework for partial volume segmentation of brain MR images," IEEE Trans. Med. Imag., vol. 18, no. 10, pp. 897-908, 2003.

[46] J. Atif, H. Khotanlou, E. Angelini, H. Duffau, and I. Bloch, "Segmentation of internal brain structures in the presence of a tumor," in MICCAI - Oncology Workshop, 2006, pp. 61-68.

[47] F. Forbes and N. Peyrard, "Hidden Markov random field model selection criteria based on mean field-like approximations," IEEE Trans. Pattern Anal. Mach. Intell., vol. 25, no. 9, pp. 1089-1101, 2003. 